Pacific

Journal of

Mathematics

\title{
STRONGLY $r$-MATRIX INDUCED TENSORS, KOSZUL COHOMOLOGY, AND ARBITRARY-DIMENSIONAL QUADRATIC POISSON COHOMOLOGY
}

Mourad Ammar, Guy Kass, Mohsen Masmoudi And Norbert Poncin 


\title{
STRONGLY $r$-MATRIX INDUCED TENSORS, KOSZUL COHOMOLOGY, AND ARBITRARY-DIMENSIONAL QUADRATIC POISSON COHOMOLOGY
}

\author{
Mourad Ammar, Guy Kass, Mohsen Masmoudi And Norbert Poncin
}

\begin{abstract}
We introduce the concept of strongly $r$-matrix induced (SRMI) Poisson structure, report on the relation of this property to the stabilizer dimension of the considered quadratic Poisson tensor, and classify the Poisson structures of the Dufour-Haraki classification (DHC) according to their membership in the family of SRMI tensors. A main result is a generic cohomological procedure for classifying SRMI Poisson structures in arbitrary dimension. This approach allows the decomposition of Poisson cohomology into, basically, a Koszul cohomology and a relative cohomology. Also we investigate this associated Koszul cohomology, highlight its tight connections with spectral theory, and reduce the computation of this main building block of Poisson cohomology to a problem of linear algebra. We apply these upshots to two structures of the DHC and provide an exhaustive description of their cohomology. We thus complete our list of data obtained in previous work, and gain fairly good insight into the structure of Poisson cohomology.
\end{abstract}

\section{Introduction}

Let $(\mathscr{L},[\cdot, \cdot])$ with $\mathscr{L}=\bigoplus_{i} \mathscr{L}^{i}$ be a graded Lie algebra (gLa). Any element with degree 1 that squares to 0 generates a differential graded Lie algebra (dgLa) $\left(\mathscr{L},[\cdot, \cdot], \partial_{\Lambda}\right)$, where $\partial_{\Lambda}:=[\Lambda, \cdot]$, and a gLa $H\left(\mathscr{L},[\cdot, \cdot], \partial_{\Lambda}\right)$ in cohomology. Depending on the initial algebra, such a 2-nilpotent degree 1 element is, say, an associative algebra structure, a Lie algebra structure, or a Poisson structure, and the associated cohomology is the adjoint Hochschild, the adjoint ChevalleyEilenberg, or the Lichnerowicz-Poisson (LP) (or simply Poisson) cohomology, respectively. Recall that the LP-dgLa is implemented by the shifted Grassmann

MSC2000: 17B56, 17B63, 55N99.

Keywords: $r$-matrix, quadratic Poisson structure, Poisson cohomology, Lichnerowicz-Poisson cohomology, Koszul cohomology, relative cohomology, spectral theory.

Ammar and Poncin were supported by grant R1F105L10. Poncin thanks the Erwin Schrödinger Institute in Vienna for hospitality and support during his visits in 2006 and 2007. 
algebra $\left(\mathscr{X}(M)[1], \wedge,[\cdot, \cdot]_{\mathrm{SN}}\right)$, with $\mathscr{X}(M)=\Gamma(\bigwedge T M)$, of polyvectors of a manifold $M$, endowed with the Schouten-Nijenhuis bracket $[\cdot, \cdot]_{\mathrm{SN}}$. The Hochschild dgLa is generated by the space of multilinear mappings of the underlying vector space endowed with the Gerstenhaber graded Lie bracket, and similarly the Chevalley-Eilenberg dgLa is generated by the space of skew-symmetric multilinear mappings of the underlying vector space endowed with the Nijenhuis-Richardson graded Lie bracket.

Alternatively, LP-cohomology can be viewed as the Lie algebroid (Lad) cohomology of the Lie algebroid $\left(T^{*} M,\{\cdot, \cdot\}, \sharp\right)$ canonically associated with an arbitrary Poisson manifold $(M, \Lambda)$. The cohomology of a $\operatorname{Lad}(E \rightarrow M, \mathbb{[} \cdot, \cdot \mathbb{1}, \rho)$, or equivalently a $Q$-structure on a supermanifold, is defined as the cohomology of the Chevalley-Eilenberg subcomplex of the representation $\rho: \Gamma(E) \rightarrow \operatorname{Der}\left(C^{\infty}(M)\right)$, made up by tensorial cochains. Algebraically, LP-cohomology is defined as the adjoint Chevalley-Eilenberg cohomology of any Poisson-Lie algebra, restricted to the cochain subspace of skew-symmetric multiderivations.

More details about Poisson cohomology can be found, say, in [Lichnerowicz 1977; Vaisman 1994].

The last few decades have seen much work on Poisson cohomology and Poisson homology, starting with [Koszul 1985; Brylinski 1988]. Problems studied include the cohomology of regular Poisson manifolds [Vaisman 1990; Xu 1992], (co)homology and resolutions [Huebschmann 1990], duality [Huebschmann 1999; Xu 1999; Evens et al. 1999], cohomology in low dimensions or specific cases [Nakanishi 1997; Ginzburg 1999; Gammella 2002; Monnier 2002b; 2002a; Roger and Vanhaecke 2002; Roytenberg 2002; Pichereau 2005], and various extensions of Poisson cohomology - for example, the cohomologies Lie algebroid, Jacobi, Nambu-Poisson, double Poisson, and graded Jacobi [de León et al. 1997; Ibáñez et al. 2001; Monnier 2001; Grabowski and Marmo 2003; de León et al. 2003; Nakanishi 2006; Pichereau and Van de Weyer 2008]. In [Masmoudi and Poncin 2007; Ammar and Poncin 2008], we suggest an approach to the cohomology of the Poisson tensors of the Dufour-Haraki classification (DHC).

Here we focus on the formal LP-cohomology associated with the quadratic Poisson tensors (QPTs) $\Lambda$ of $\mathbb{R}^{n}$ that read as real linear combinations

$$
\Lambda=\sum_{i<j} \alpha^{i j} Y_{i} \wedge Y_{j}=: \sum_{i<j} \alpha^{i j} Y_{i j} \quad \text { for } \alpha^{i j} \in \mathbb{R}
$$

of the wedge products of $n$ commuting linear vector fields $Y_{1}, \ldots, Y_{n}$, such that $Y_{1} \wedge \cdots \wedge Y_{n}=: Y_{1 \ldots n} \neq 0$. Let us recall that "formal" means that we substitute the space $\mathbb{R} \llbracket x_{1}, \ldots, x_{n} \rrbracket \otimes \wedge \mathbb{R}^{n}$ of multivectors with coefficients in the formal series for the usual Poisson cochain space $\mathscr{\mathscr { L }}\left(\mathbb{R}^{n}\right)=C^{\infty}\left(\mathbb{R}^{n}\right) \otimes \bigwedge \mathbb{R}^{n}$. Furthermore, the 
reader may think about QPTs of type (1-1) as QPTs implemented by a classical $r$-matrix in their stabilizer for the canonical matrix action.

Hence, in Section 2, we are interested in characterizing the QPTs that are images of a classical $r$-matrix. We show that a QPT is induced by an $r$-matrix if the dimension of its stabilizer is large enough; more precisely, we prove that if the stabilizer of a given QPT $\Lambda$ of $\mathbb{R}^{n}$ contains $n$ commuting linear vector fields $Y_{i}$ such that $Y_{1 \ldots n} \neq 0$, then $\Lambda$ is implemented by an $r$-matrix in its stabilizer; see Corollary 2. We refer to such tensors as strongly $r$-matrix induced (SRMI) structures and show that any structure of the DHC decomposes into the sum of a maximal SRMI structure and a small compatible (mostly exact) Poisson tensor; see Theorem 4. This decomposition is the foundation of our cohomological techniques proposed in [Masmoudi and Poncin 2007; Ammar and Poncin 2008]. This splitting is in some sense opposite to the one proved in [Liu and Xu 1992], which incorporates the largest possible part of the Poisson tensor into the exact term.

Masmoudi and Poncin [2007] developed a cohomological method in Euclidean three-space that greatly simplified LP-cohomology computations for the SRMI structures of the Dufour-Haraki classification. Section 3 extends this procedure to arbitrary-dimensional vector spaces. In Theorem 10, we inject the space $\mathscr{R}$ of "real" LP-cochains (formal multivector fields) into a larger space $\mathscr{P}$ of "potential" cochains. In Theorems 13 and 15, we identify the natural extension to $\mathscr{P}$ of the LPdifferential as the Koszul differential associated with $n$ commuting endomorphisms

$$
X_{i}-\left(\operatorname{div} X_{i}\right) \text { id, } \quad \text { where } X_{i}=\sum_{j} \alpha^{i j} Y_{j} \text { and } \alpha^{j i}=-\alpha^{i j} \text {, }
$$

of the space made up by the polynomials on $\mathbb{R}^{n}$ with some fixed homogeneous degree. We then choose a space $\mathscr{S}$ supplementary to $\mathscr{R}$ in $\mathscr{P}$ and show that the LPdifferential induces a differential on $\mathscr{Y}$. Eventually, we end up with a short exact sequence of differential spaces and an exact triangle in cohomology. Theorem 16 shows that LP-cohomology ( $\mathscr{R}$-cohomology) reduces, essentially, to Koszul cohomology ( $\mathscr{P}$-cohomology) and a relative cohomology ( $\mathscr{P}$-cohomology).

To take advantage of these results, we investigate in Section 4 the Koszul cohomology associated to $n$ commuting linear operators on a finite-dimensional complex vector space. We prove a homotopy-type formula in Proposition 19 andusing spectral properties - show in Theorem 20 and Corollary 21 that the Koszul cohomology is located inside a primary subspace of the corresponding commuting endomorphisms.

In Section 5, we apply this result to gain insight into the structure of the Koszul cohomology implemented by SRMI tensors, and show that to compute this central part of Poisson cohomology it basically suffices to solve triangular systems of linear equations. 
We conclude Section 5 by providing a full description of the LP-cohomology spaces of structures $\Lambda_{3}$ and $\Lambda_{9}$ of the Dufour-Haraki classification.

\section{Characterization of strongly $\boldsymbol{r}$-matrix induced Poisson structures}

Stabilizer dimension and r-matrix generation. Poisson structures implemented by an $r$-matrix are of interest, for example in deformation quantization, especially in view of Drinfeld's method. We next report on an idea for generating quadratic Poisson tensors by classical $r$-matrices.

Set $G=\operatorname{GL}(n, \mathbb{R})$ and $\mathfrak{g}=\mathfrak{g l}(n, \mathbb{R})$. The Lie algebra isomorphism between $\mathfrak{g}$ and the algebra $\mathscr{X}_{0}^{1}\left(\mathbb{R}^{n}\right)$ of linear vector fields extends to a Grassmann algebra and a graded Poisson-Lie algebra homomorphism $J: \bigwedge \mathfrak{g} \rightarrow \bigoplus_{k}\left(9^{k} \mathbb{R}^{n *} \otimes \bigwedge^{k} \mathbb{R}^{n}\right)$. It is known that its restriction

$$
J^{k}: \bigwedge^{k} \mathfrak{g} \rightarrow \mathscr{S}^{k} \mathbb{R}^{n *} \otimes \bigwedge^{k} \mathbb{R}^{n}
$$

is onto, but has a nontrivial kernel if $k, n \geq 2$. In particular,

$$
J^{3}[r, r]_{\mathrm{SN}}=\left[J^{2} r, J^{2} r\right]_{\mathrm{SN}} \quad \text { for } r \in \mathfrak{g} \wedge \mathfrak{g},
$$

where $[\cdot, \cdot]_{\mathrm{SN}}$ is the Schouten-Nijenhuis bracket. It is still an open problem to characterize the quadratic Poisson structures that are implemented by a classical $r$-matrix, that is, a bimatrix $r \in \mathfrak{g} \wedge \mathfrak{g}$ satisfying the classical Yang-Baxter equation $[r, r]_{\mathrm{SN}}=0$.

Quadratic Poisson tensors $\Lambda_{1}$ and $\Lambda_{2}$ are equivalent if and only if there is $A \in G$ such that $A_{*} \Lambda_{1}=\Lambda_{2}$, where $*$ denotes the standard action of $G$ on tensors of $\mathbb{R}^{n}$. Since $J^{2}$ is a $G$-module homomorphism, that is,

$$
A_{*}\left(J^{2} r\right)=J^{2}(\operatorname{Ad}(A) r) \text { for } A \in G \text { and } r \in \mathfrak{g} \wedge \mathfrak{g},
$$

the $G$-orbit of a quadratic Poisson structure $\Lambda=J^{2} r$ is the pointwise $J^{2}$-image of the $G$-orbit of $r$. Furthermore, the representation Ad acts by graded Lie algebra homomorphisms, that is,

$$
\operatorname{Ad}(A)[r, r]_{\mathrm{SN}}=[\operatorname{Ad}(A) r, \operatorname{Ad}(A) r]_{\mathrm{SN}} .
$$

Hence, if $\Lambda=J^{2} r$, where $r$ is a classical $r$-matrix, the orbit of this quadratic Poisson tensor consists of $r$-matrix induced structures.

Any quadratic Poisson tensor $\Lambda$ is implemented by bimatrices $r \in \mathfrak{g} \wedge \mathfrak{g}$. To determine whether the $G$-orbit $O_{\Lambda}$ of this tensor is generated by $r$-matrices, we look at the preimage $\left(J^{2}\right)^{-1}\left(O_{\Lambda}\right)=\bigcup_{r \in\left(J^{2}\right)^{-1} \Lambda} O_{r}$, composed of the $G$-orbits $O_{r}$ of all the bimatrices $r$ that are mapped on $\Lambda$ by $J^{2}$. We claim that the bigger the chance that a fiber of this bundle is located inside $r$-matrices, the smaller is $O_{\Lambda}$. 
In other words, the dimension of the isotropy Lie group $G_{\Lambda}$ of $\Lambda$, or of its Lie algebra, the stabilizer

$$
\mathfrak{g}_{\Lambda}:=\left\{a \in \mathfrak{g}:[\Lambda, J a]_{\mathrm{SN}}=0\right\}
$$

of $\Lambda$ for the corresponding infinitesimal action, should be big enough. For example, in $\mathbb{R}^{3}$ the Poisson tensor $\Lambda=\left(x_{1}^{2}+x_{2} x_{3}\right) \partial_{23}$, where $\partial_{23}:=\partial_{2} \wedge \partial_{3}$ and $\partial_{i}:=\partial / \partial_{x_{i}}$, is not $r$-matrix induced (see [Manchon et al. 2002]) and the dimension of its stabilizer is $\operatorname{dim} \mathfrak{g}_{\Lambda}=2$. More evidence comes from the corollary of the following theorem:

Theorem 1. Let $\Lambda$ be an analytic Poisson tensor of $\mathbb{R}^{n}$. If its stabilizer contains $n$ commuting analytic vector fields $Y_{i}$ for $i \in\{1, \ldots, n\}$ such that $Y_{1} \wedge \cdots \wedge Y_{n} \neq 0$, then there exist constants $\alpha^{k l} \in \mathbb{R}$ such that $\Lambda=\sum_{k<l} \alpha^{k l} Y_{k} \wedge Y_{l}$.

Proof. Since $Y_{1} \wedge \cdots \wedge Y_{n} \neq 0$, there exists an open subset $O$ of $\mathbb{R}^{n}$ such that

$$
\Lambda=\sum_{k<l} \alpha^{k l}(x) Y_{k} \wedge Y_{l} \quad \text { in } O
$$

for some local functions $\alpha^{k l}=\alpha^{k l}(x)$. Since for any $i \in\{1, \ldots, n\}$, we have

$$
0=\left[Y_{i}, \Lambda\right]_{\mathrm{SN}}=\sum_{k<l} Y_{i}\left(\alpha^{k l}\right) Y_{k} \wedge Y_{l} \quad \text { in } O,
$$

the $\alpha^{k l}$ are constant in $O$; the theorem follows by analytic continuation.

Corollary 2. Let $\Lambda$ be a quadratic Poisson tensor of $\mathbb{R}^{n}$. If its stabilizer $\mathfrak{g}_{\Lambda}$ contains $n$ commuting linear vector fields $Y_{i}$ for $i \in\{1, \ldots, n\}$ such that $Y_{1} \wedge \cdots \wedge Y_{n}$ does not vanish, then $\Lambda$ is implemented by a classical $r$-matrix that belongs to the stabilizer, that is, $\Lambda=J^{2} a$, where $[a, a]_{\mathrm{SN}}=0$ and $a \in \mathfrak{g}_{\Lambda} \wedge \mathfrak{g}_{\Lambda}$.

Definition 3. If $\Lambda$ is a quadratic Poisson structure implemented by a classical $r$-matrix $r \in \mathfrak{g}_{\Lambda} \wedge \mathfrak{g}_{\Lambda}$, we call $\Lambda$ a strongly $r$-matrix induced (SRMI) tensor.

Classification theorem in Euclidean three-space. Two concepts of exact Poisson structure - which are closely related to two special cohomology classes are used below. Let $\Lambda$ be a Poisson tensor on a smooth manifold $M$ oriented by a volume element $\Omega$. We say that $\Lambda$, which is of course a LP-2-cocycle, is Lichnerowicz-Poisson-exact or LP-exact if

$$
\Lambda=[\Lambda, X]_{\mathrm{SN}} \text { for some } X \in \mathscr{L}^{1}(M) .
$$

The vector field $X$ is called the Liouville vector field and the cohomology class of $\Lambda$ is the obstruction to infinitesimal rescaling of $\Lambda$. We call $\Lambda$ Koszul-exact or $\mathrm{K}$-exact if

$$
\Lambda=\delta(T) \quad \text { for some } T \in \mathscr{X}^{3}(M) \text {. }
$$


Here, the operator $\delta:=\phi^{-1} \circ d \circ \phi$ is the pullback of the de Rham differential $d$ by the canonical vector space isomorphism $\phi:=i_{(\cdot)} \Omega$. Although introduced earlier, the generalized divergence $\delta$ defined by $\delta(X)=\operatorname{div}_{\Omega} X$ for $X \in \mathscr{L}^{1}(M)$ is usually attributed to J.-L. Koszul. The curl vector field $K(\Lambda):=\delta(\Lambda)$ of $\Lambda^{1}$ is an LP-1cocycle. $K(\Lambda)$ maps a function to the divergence of its Hamiltonian vector field. The cohomology class of $K(\Lambda)$ is the well-known modular class of $\Lambda$. This class is independent of $\Omega$, is the obstruction to existence on $M$ of a measure preserved by all Poisson automorphisms, and is relevant in the classification of Poisson structures [Dufour and Haraki 1991; Grabowski et al. 1993; Liu and Xu 1992] and in Poincaré duality [Evens et al. 1999; Ibáñez et al. 2001]. In $\mathbb{R}^{n}$ with $n \geq 3$, a Poisson tensor $\Lambda$ is $\mathrm{K}$-exact if and only if it is irrotational, that is, $K(\Lambda)=0$, and in $\mathbb{R}^{3}$, K-exact means function-induced, that is,

$$
\Lambda=\Pi_{f}:=\partial_{1} f \partial_{23}+\partial_{2} f \partial_{31}+\partial_{3} f \partial_{12} \text { for } f \in C^{\infty}\left(\mathbb{R}^{3}\right) .
$$

The K-exact quadratic Poisson tensors $\Pi_{p}$ of $\mathbb{R}^{3}$, that is, the K-exact Poisson structures induced by a homogeneous polynomial $p \in \mathscr{Y}^{3} \mathbb{R}^{3 *}$, represent class 14 of the Dufour-Haraki classification. The cohomology of this class has been studied by Pichereau [2005] (actually Pichereau deals with structures $\Pi_{p}$ implemented by a weight-homogeneous polynomial $p$ with an isolated singularity). Hence, we will not examine class 14 here.

Recall that two Poisson tensors $\Lambda_{1}$ and $\Lambda_{2}$ are compatible if their sum is again a Poisson structure, that is, if $\left[\Lambda_{1}, \Lambda_{2}\right]_{\mathrm{SN}}=0$.

The next theorem classifies the quadratic Poisson tensors according to their strongly $r$-matrix induced structure. It also shows that any such tensor is the sum of a "maximal" strongly $r$-matrix induced tensor and a "small" compatible Poisson structure. The classification makes available the cohomological technique used in [Masmoudi and Poncin 2007], while the splitting ${ }^{2}$ is relevant to cohomological approach of [Ammar and Poncin 2008].

Denote the canonical coordinates of $\mathbb{R}^{3}$ by $x, y, z$ and by $x_{1}, x_{2}, x_{3}$; denote the corresponding partial derivatives by $\partial_{1}, \partial_{2}, \partial_{3}$. Let $\partial_{i j}=\partial_{i} \wedge \partial_{j}$.

Theorem 4. Let $a, b, c \in \mathbb{R}$, and let $\Lambda_{i}$ for $i \in\{1, \ldots, 13\}$ be the quadratic Poisson tensors of the Dufour-Haraki classification [1991].

If $\operatorname{dim} \mathfrak{g}_{\Lambda}>3$ (where the subscript $i$ is omitted), there are mutually commuting linear vector fields $Y_{1}, Y_{2}, Y_{3}$ such that

$$
\Lambda=\alpha Y_{23}+\beta Y_{31}+\gamma Y_{12}, \quad \text { where } \alpha, \beta, \gamma \in \mathbb{R},
$$

\footnotetext{
${ }^{1}$ If $\Omega$ is the standard volume of $\mathbb{R}^{3}$ and $\Lambda$ is identified with a vector field $\vec{\Lambda}$ of $\mathbb{R}^{3}$, then $K(\Lambda)$ coincides with the standard curl $\vec{\nabla} \wedge \vec{\Lambda}$.

${ }^{2}$ This splitting differs from the decomposition used in [Liu and Xu 1992] in that we incorporate as much structure as possible into the strongly induced term.
} 
so that $\Lambda$ is strongly $r$-matrix induced (SRMI), that is, implemented by a classical $r$-matrix in $\mathfrak{g}_{\Lambda} \wedge \mathfrak{g}_{\Lambda}$. In the following classification of the quadratic Poisson tensors by the SRMI property, we decompose each non-SRMI tensor into the sum of a maximal SRMI structure and a smaller compatible quadratic Poisson tensor.

- Set $Y_{1}=x \partial_{1}, Y_{2}=y \partial_{2}$ and $Y_{3}=z \partial_{3}$.

(1) $\Lambda_{1}=a y z \partial_{23}+b x z \partial_{31}+c x y \partial_{12}$ is SRMI for all values of the parameters $a, b$ and $c$, and decomposes as $\Lambda_{1}=a Y_{23}+b Y_{31}+c Y_{12}$.

(2) $\Lambda_{4}=a y z \partial_{23}+a x z \partial_{31}+\left(b x y+z^{2}\right) \partial_{12}$ is SRMI if and only if $a$ and $b$ are both zero. We have $\Lambda_{4}=a\left(Y_{23}+Y_{31}\right)+b Y_{12}+\frac{1}{3} \Pi_{z^{3}}$.

- Set $Y_{1}=x \partial_{1}+y \partial_{2}, Y_{2}=x \partial_{2}-y \partial_{1}$ and $Y_{3}=z \partial_{3}$.

(1) $\Lambda_{2}=(2 a x-b y) z \partial_{23}+(b x+2 a y) z \partial_{31}+a\left(x^{2}+y^{2}\right) \partial_{12}$ is SRMI for any $a$ and $b$. We have $\Lambda_{2}=2 a Y_{23}+b Y_{31}+a Y_{12}$.

(2) $\Lambda_{7}=((2 a+c) x-b y) z \partial_{23}+(b x+(2 a+c) y) z \partial_{31}+a\left(x^{2}+y^{2}\right) \partial_{12}$ is SRMI for all $a, b, c$. We have $\Lambda_{7}=(2 a+c) Y_{23}+b Y_{31}+a Y_{12}$.

(3) $\Lambda_{8}=a x z \partial_{23}+a y z \partial_{31}+\left(\frac{1}{2}(a+b)\left(x^{2}+y^{2}\right) \pm z^{2}\right) \partial_{12}$ is SRMI if and only if $a$ and $b$ are both zero. We have

$$
\Lambda_{8}=a Y_{23}+\frac{1}{2}(a+b) Y_{12} \pm \frac{1}{3} \Pi_{z^{3}} .
$$

- Set $Y_{1}=x \partial_{1}+y \partial_{2}, Y_{2}=x \partial_{2}$ and $Y_{3}=z \partial_{3}$.

(1) $\Lambda_{3}=(2 x-a y) z \partial_{23}+a x z \partial_{31}+x^{2} \partial_{12}$ is SRMI for any $a$, and we have $\Lambda_{3}=2 Y_{23}+a Y_{31}+Y_{12}$.

(2) $\Lambda_{5}=((2 a+1) x+y) z \partial_{23}-x z \partial_{31}+a x^{2} \partial_{12}$ is SRMI for any $a \neq-1 / 2$. We have $\Lambda_{5}=(2 a+1) Y_{23}-Y_{31}+a Y_{12}$.

(3) $\Lambda_{6}=a y z \partial_{23}-a x z \partial_{31}-\frac{1}{2} x^{2} \partial_{12}$ is SRMI for any $a$. The decomposition is $\Lambda_{6}=-a Y_{31}-\frac{1}{2} Y_{12}$.

- Set $Y_{1}=\mathscr{E}:=x \partial_{1}+y \partial_{2}+z \partial_{3}, Y_{2}=x \partial_{2}+y \partial_{3}$ and $Y_{3}=x \partial_{3}$.

(1) $\Lambda_{9}=\left(a x^{2}-\frac{1}{3} y^{2}+\frac{1}{3} x z\right) \partial_{23}+\frac{1}{3} x y \partial_{31}-\frac{1}{3} x^{2} \partial_{12}$ is SRMI for any $a$. We have $\Lambda_{9}=a Y_{23}-\frac{1}{3} Y_{12}$.

(2) $\Lambda_{10}=\left(a y^{2}-(4 a+1) x z\right) \partial_{23}+(2 a+1) x y \partial_{31}-(2 a+1) x^{2} \partial_{12}$ is SRMI if and only if $a=-1 / 3$. We have $\Lambda_{10}=-(2 a+1) Y_{12}+(3 a+1)\left(y^{2}-2 x z\right) \partial_{23}$.

- Set $Y_{1}=\mathscr{E}, Y_{2}=x \partial_{2}$ and $Y_{3}=(a x+(3 b+1) z) \partial_{3}$.

(1) Set $a=0$. Then $\Lambda_{11}=(2 b+1) x z \partial_{23}+\left(b x^{2}+c z^{2}\right) \partial_{12}$ is SRMI if and only if $c=0$. We have $\Lambda_{11}=Y_{23}+b Y_{12}+\frac{1}{3} c \Pi_{z^{3}}$.

(2) Set $a=1$. Then $\Lambda_{12}=\left(x^{2}+(2 b+1) x z\right) \partial_{23}+\left(b x^{2}+c z^{2}\right) \partial_{12}$ is SRMI if and only if $c=0$. We have $\Lambda_{12}=Y_{23}+b Y_{12}+\frac{1}{3} c \Pi_{z^{3}}$.

(3) $\Lambda_{13}=\left(a x^{2}+(2 b+1) x z+z^{2}\right) \partial_{23}+\left(b x^{2}+c z^{2}+2 x z\right) \partial_{12}$ is not SRMI for any $a, b, c$. We have $\Lambda_{13}=Y_{23}+b Y_{12}+\Pi_{c z^{3} / 3+x z^{2}}$. 
Proof. The basic fields $Y_{1}, Y_{2}, Y_{3}$ have been read in the stabilizers of the considered Poisson tensors, but for brevity we omit the stabilizer computations. Indeed, once the vector fields $Y_{i}$ are specified, it is easily checked that, in the SRMI cases, they satisfy the assumptions of Theorem 1 . Thus the corresponding Poisson structures are actually SRMI tensors. To show that a quadratic Poisson structure $\Lambda$ is not SRMI, it suffices to prove that $\Lambda \notin J^{2}\left(\mathfrak{g}_{\Lambda} \wedge \mathfrak{g}_{\Lambda}\right)$, which we will do below.

All the decompositions above can be directly verified. In most instances, the twist is obviously Poisson, so that compatibility follows. In the case of $\Lambda_{10}$, the twist $\Lambda_{10, \mathrm{II}}=\left(y^{2}-2 x z\right) \partial_{23}$ is a non-K-exact Poisson structure, which follows directly from the fact that $K\left(\Lambda_{10, \mathrm{II}}\right)=\vec{\nabla} \wedge \vec{\Lambda}_{10, \mathrm{II}}=-2 x \partial_{2}-2 y \partial_{3} \neq 0$ and the formula $[P, Q]_{\mathrm{SN}}=(-1)^{p} D(P \wedge Q)-D(P) \wedge Q-(-1)^{p} P \wedge D(Q)$ for $P \in \mathscr{L}^{p}(M)$ and $Q \in \mathscr{X}^{q}(M)$. The main part of this proof will make it obvious why we require that $\operatorname{dim} \mathfrak{g}_{\Lambda}>3$.

Denote by $E_{i j}$ for $i, j \in\{1,2,3\}$ the canonical basis of $\mathfrak{g l}(3, \mathbb{R})$.

- If $(a, b) \neq(0,0)$, stabilizer $\mathfrak{g}_{\Lambda_{4}}$ and the image $J^{2}\left(\mathfrak{g}_{\Lambda_{4}} \wedge \mathfrak{g}_{\Lambda_{4}}\right)$ are generated by $\left(\frac{1}{2} E_{11}+E_{22}, \frac{1}{2} E_{11}+E_{33}\right)$ and $y z \partial_{23}-\frac{1}{2} x z \partial_{31}-\frac{1}{2} x y \partial_{12}$, respectively. Hence $\Lambda_{4}$ is not SRMI.

- If $(a, b) \neq(0,0)$, the generators of $\mathfrak{g}_{\Lambda_{8}}$ and $J^{2}\left(\mathfrak{g}_{\Lambda_{8}} \wedge \mathfrak{g}_{\Lambda_{8}}\right)$ are

$$
\left(E_{11}+E_{22}+E_{33}, E_{12}-E_{21}\right) \text { and }-x z \partial_{23}-y z \partial_{31}+\left(x^{2}+y^{2}\right) \partial_{12} .
$$

So $\Lambda_{8}$ is not SRMI.

- If $a \neq-1 / 3$, the generators in the case of $\Lambda_{10}$ are

$$
\left(E_{11}+E_{22}+E_{33}, E_{12}+E_{23}\right) \text { and }\left(y^{2}-x z\right) \partial_{23}-x y \partial_{31}+x^{2} \partial_{12} .
$$

- For the cases of $\Lambda_{11}$ and $\Lambda_{12}$ with $c \neq 0$, and the case $\Lambda_{13}$, the generators are

$$
\left(E_{11}+E_{22}+E_{33}, E_{12}, E_{32}\right) \text { and }-x z \partial_{23}+x^{2} \partial_{12}, z^{2} \partial_{23}-x z \partial_{12} .
$$

Remarks. - In the cases $\Lambda_{1}, \Lambda_{2}$ and $\Lambda_{3}$, with $c \neq 0$ in the latter two, the dimension of the stabilizer is $\operatorname{dim} \mathfrak{g}_{\Lambda}=3$, whereas $J \mathfrak{g}_{\Lambda} \wedge J \mathfrak{g}_{\Lambda} \wedge J \mathfrak{g}_{\Lambda}=\{0\}$. Hence, if the dimension of the stabilizer coincides with the dimension of the space, the Poisson structure is not necessarily a SRMI tensor.

- For $\Lambda_{10}$, the decomposition proved in [Liu and Xu 1992] yields

$$
\Lambda_{10}=-\frac{1}{3} Y_{12}+\Pi_{c z^{3} / 3+x z^{2}+(b+1 / 3) x^{2} z+a x^{3} / 3} .
$$

\section{Poisson cohomology of quadratic structures in a finite-dimensional vector space}

Koszul homology and cohomology. Let $\bigwedge=\bigwedge_{n}\langle\vec{\eta}\rangle$ be the Grassmann algebra on $n \in \mathbb{N}_{0}$ with generators $\vec{\eta}=\left(\eta_{1}, \ldots, \eta_{n}\right)$, that is, the algebra over a field $\mathbb{F}$ (here $\mathbb{R}$ 
or $\mathbb{C}$ ) of characteristic 0 generated by $\eta_{1}, \ldots, \eta_{n}$ and subject to the anticommutation relations $\eta_{k} \eta_{\ell}+\eta_{\ell} \eta_{k}=0$ for $k, \ell \in\{1, \ldots, n\}$. Set $\Lambda=\bigoplus_{p=0}^{n} \bigwedge^{p}$, with obvious notations, and let $\vec{h}=\left(h_{1}, \ldots, h_{n}\right)$ be dual generators defined by $i_{h_{k}} \eta_{\ell}=\delta_{k \ell}$. We also need the creation operator $e_{\eta_{k}}: \bigwedge \rightarrow \bigwedge, \omega \mapsto \eta_{k} \omega$ and the annihilation operator $i_{h_{k}}: \bigwedge \rightarrow \bigwedge, \omega \mapsto i_{h_{k}} \omega$, where the interior product is defined as usual. Finally, we denote by $E$ a vector space over $\mathbb{F}$ and by $\vec{X}=\left(X_{1}, \ldots, X_{n}\right)$ an $n$-tuple of commuting linear operators on $E$.

Definition 5. The Koszul chain complex (or $K_{*}$-complex) $K_{*}(\vec{X}, E)$ associated with $\vec{X}$ on $E$ is the complex

$$
0 \rightarrow E \otimes_{\mathbb{F}} \bigwedge^{n} \rightarrow E \otimes_{\mathbb{F}} \bigwedge^{n-1} \rightarrow \cdots \rightarrow E \otimes_{\mathbb{F}} \bigwedge^{1} \rightarrow E \rightarrow 0
$$

with differential $\kappa_{\vec{X}}=\sum_{k=1}^{n} X_{k} \otimes i_{h_{k}}$. We denote by $K H_{*}(\vec{X}, E)$ the corresponding Koszul homology group.

Definition 6. The Koszul cochain complex (or $K^{*}$-complex) $K^{*}(\vec{X}, E)$ associated with $\vec{X}$ on $E$ is the complex

$$
0 \rightarrow E \rightarrow E \otimes_{\mathbb{F}} \bigwedge^{1} \rightarrow \ldots \rightarrow E \otimes_{\mathbb{F}} \bigwedge^{n-1} \rightarrow E \otimes_{\mathbb{F}} \bigwedge^{n} \rightarrow 0
$$

with differential $\mathscr{K}_{\vec{X}}=\sum_{k=1}^{n} X_{k} \otimes e_{\eta_{k}}$. We denote by $K H^{*}(\vec{X}, E)$ the corresponding Koszul cohomology group.

Since the $X_{k}$ commute, the anticommutativity of the $i_{h_{k}}$ and the $e_{\eta_{k}}$ imply that $\kappa_{\vec{X}} \kappa_{\vec{X}}=0$ and $\mathscr{K}_{\vec{X}} \mathcal{K}_{\vec{X}}=0$, respectively. See [Koszul 1950; 1994].

Example 7. Let $\mathbb{F}=\mathbb{R}$ and $E=C^{\infty}\left(\mathbb{R}^{3}\right)$. If we choose $\eta_{k}=d x_{k}$ and $X_{k}=\partial_{k}$, the $K^{*}$-complex is the de Rham complex $\left(\Omega\left(\mathbb{R}^{3}\right), d\right)$. With $\eta_{k}=\partial_{k}=\partial_{x_{k}}$ and $h_{k}=d x_{k}$, the $K_{*}$-complex is the dual de Rham complex $\left(\mathscr{X}\left(\mathbb{R}^{3}\right), \delta\right)$.

If we identify the subspaces $\Omega^{k}\left(\mathbb{R}^{3}\right)$ of homogeneous forms with the corresponding spaces of components $E, E^{3}, E^{3}$ and $E$, this $K^{*}$-complex reads

$$
0 \rightarrow E \stackrel{\mathscr{K}=\vec{\nabla}(\cdot)}{\longrightarrow} E^{3} \stackrel{\mathscr{K}=\vec{\nabla} \wedge(\cdot)}{\longrightarrow} E^{3} \stackrel{\mathscr{K}=\vec{\nabla} \cdot(\cdot)}{\longrightarrow} E \rightarrow 0 .
$$

Example 8. Let $\mathbb{F}=\mathbb{R}$ and $E=\mathscr{Y} \mathbb{R}^{3 *}=\mathbb{R}\left[x_{1}, x_{2}, x_{3}\right]$. For $k \in\{1,2,3\}$, let $\eta_{k}=\partial_{k}$, $X_{k}=\mathfrak{m}_{P_{k}}, P_{k} \in E^{d_{k}}$ and $d_{k} \in \mathbb{N}$, where $\mathfrak{m}_{P_{k}}: E \rightarrow E, Q \mapsto P_{k} Q$. Then the chain spaces of the $K_{*}$-complex are the spaces of homogeneous polyvector fields on $\mathbb{R}^{3}$ with polynomial coefficients, and by identifying these with the corresponding spaces $E, E^{3}, E^{3}$ and $E$ of components, we can write this $K_{*}$-complex in the form

$$
0 \rightarrow E \stackrel{\kappa=(\cdot) \vec{P}}{\longrightarrow} E^{3} \stackrel{\kappa=(\cdot) \wedge \vec{P}}{\longrightarrow} E^{3} \stackrel{\kappa=(\cdot) \cdot \vec{P}}{\longrightarrow} E \rightarrow 0 .
$$

Remarks. First, the Koszul cohomology and homology complexes of Example 7 are exact, except that $K H^{0}\left(\vec{\partial}, C^{\infty}\left(\mathbb{R}^{3}\right)\right) \simeq K H_{3}\left(\vec{\partial}, C^{\infty}\left(\mathbb{R}^{3}\right)\right) \simeq \mathbb{R}$. 
Second, recall that an $R$-regular sequence on a module $M$ over a commutative unit ring $R$ is a sequence $\left(r_{1}, \ldots, r_{d}\right) \in R^{d}$ such that $r_{k}$ is not a zero divisor on the quotient $M /\left\langle r_{1}, \ldots, r_{k-1}\right\rangle M$ for $k \in\{1, \ldots, d\}$, and $M /\left\langle r_{1}, \ldots, r_{d}\right\rangle M \neq 0$. In particular, $x_{1}, \ldots, x_{d}$ is a (maximal length) regular sequence on the polynomial ring $R=\mathbb{F}\left[x_{1}, \ldots, x_{d}\right]$, so that this ring has depth $d$.

It is well known that the $K_{*}$-complex described in Example 8 is exact, except for surjectivity of $\kappa=(\cdot) \cdot \vec{P}$, if the vector $\vec{P}=\left(P_{1}, P_{2}, P_{3}\right)$ is regular on $\mathbb{R}\left[x_{1}, x_{2}, x_{3}\right]$. If $\vec{P}=\vec{\nabla} p$ for $p$ a homogeneous polynomial with an isolated singularity at the origin, then $\vec{P}$ is regular; see [Pichereau 2005].

Poisson cohomology in dimension 3. Set $E:=C^{\infty}\left(\mathbb{R}^{3}\right)$ and again identify the spaces of homogeneous multivector fields in $\mathbb{R}^{3}$ with the corresponding component spaces: $\mathscr{L}^{0}\left(\mathbb{R}^{3}\right) \simeq \mathscr{X}^{3}\left(\mathbb{R}^{3}\right) \simeq E$ and $\mathscr{X}^{1}\left(\mathbb{R}^{3}\right) \simeq \mathscr{X}^{2}\left(\mathbb{R}^{3}\right) \simeq E^{3}$.

Let $\vec{\Lambda}=\left(\Lambda_{1}, \Lambda_{2}, \Lambda_{3}\right) \in E^{3}$ be a Poisson tensor, and let $f \in E, \vec{X} \in E^{3}, \vec{B} \in E^{3}$ and $T \in E$ be a 0-, 1-, 2-, and 3-cochain of the LP-complex. By straightforward computations, we get formulas for the LP-coboundary operator $\partial_{\vec{\Lambda}}$ :

$$
\begin{aligned}
& \partial_{\vec{\Lambda}}^{0} f=\vec{\nabla} f \wedge \vec{\Lambda}, \\
& \partial_{\vec{\Lambda}}^{1} \vec{X}=(\vec{\nabla} \cdot \vec{X}) \vec{\Lambda}-\vec{\nabla}(\vec{X} \cdot \vec{\Lambda})+\vec{X} \wedge(\vec{\nabla} \wedge \vec{\Lambda}), \\
& \partial_{\vec{\Lambda}}^{2} \vec{B}=-(\vec{\nabla} \wedge \vec{B}) \cdot \vec{\Lambda}-\vec{B} \cdot(\vec{\nabla} \wedge \vec{\Lambda}), \\
& \partial_{\vec{\Lambda}}^{3} T=0 .
\end{aligned}
$$

Recall the differential $\mathscr{K}$ from (3-1), and let $\kappa^{\prime}$ and $\kappa^{\prime \prime}$ be the differential in (3-2) when $\vec{P}=\vec{\Lambda}$ and $\vec{P}=\vec{\nabla} \wedge \vec{\Lambda}$, respectively. Then

$$
\begin{array}{ll}
\partial_{\vec{\Lambda}}^{0}=\kappa^{\prime} \mathscr{K}, & \\
\partial_{\vec{\Lambda}}^{1}=\kappa^{\prime} \mathscr{K}-\mathscr{K} \kappa^{\prime}+\kappa^{\prime \prime}, & \partial_{\vec{\Lambda}}^{2}=-\kappa^{\prime} \mathscr{K}-\kappa^{\prime \prime}, \\
& \partial_{\vec{\Lambda}}^{3}=0 .
\end{array}
$$

Again, this paper investigates only quadratic Poisson tensors and polynomial (or formal) LP-cochains. If the structure $\vec{\Lambda}$ is $K$-exact, that is, in view of notations due to the elimination of the module basis of multivector fields, $\vec{\Lambda}=\vec{\nabla} p$ for $p \in \mathscr{G}^{3} \mathbb{R}^{3 *}$ if and only if $\vec{\nabla} \wedge \vec{\Lambda}=0$, homology operator $\kappa^{\prime \prime}$ vanishes. If, moreover, $p$ has an isolated singularity, the $K^{*}$-complex associated with $\mathscr{K}$ is exact up to injectivity of $\mathscr{K}=\vec{\nabla}(\cdot)$, and the $K_{*}$-complex associated with $\kappa^{\prime}$ is acyclic (see above) up to surjectivity of $\kappa^{\prime}=(\cdot) \cdot \vec{\Lambda}$. Pichereau [2005] computed the LP-cohomology for a weight-homogeneous polynomial $p$ with an isolated singularity.

Next we describe a generic cohomological technique for SRMI Poisson tensors in a finite-dimensional vector space. This approach extends (3-3) to dimension $n$ and also reduces the LP-coboundary operator $\partial_{\Lambda}$ to a single Koszul differential. 


\section{Poisson cohomology in dimension $n$.}

Definition 9. Let $Y_{i}=\sum_{r} \ell_{i r} \partial_{r}$ be $n$ linear vector fields in $\mathbb{R}^{n}$. Set

$$
\begin{aligned}
& \mathscr{R}=\bigoplus_{p=0}^{n} \mathscr{R}^{p}=\bigoplus_{p=0}^{n} \mathbb{R} \llbracket x_{1}, \ldots, x_{n} \rrbracket \otimes \bigwedge_{n}^{p}\langle\vec{\partial}\rangle, \\
& \mathscr{P}=\bigoplus_{p=0}^{n} \mathscr{P}^{p}=D^{-1} \bigoplus_{p=0}^{n} \mathbb{R} \llbracket x_{1}, \ldots, x_{n} \rrbracket \otimes \bigwedge_{n}^{p}\langle\vec{Y}\rangle,
\end{aligned}
$$

where $D=\operatorname{det} \ell$ and $\bigwedge_{n}^{p}\langle\vec{\partial}\rangle$ and $\bigwedge_{n}^{p}\langle\vec{Y}\rangle$ are the terms of degree $p$ of the Grassmann algebras on generators $\vec{\partial}=\left(\partial_{1}, \ldots, \partial_{n}\right)$ and $\vec{Y}=\left(Y_{1}, \ldots, Y_{n}\right)$, respectively. The spaces $\mathscr{R}$ and $\mathscr{P}$ are respectively the space of real and potential formal LP-cochains.

For $\boldsymbol{i}=\left(i_{1}, \ldots, i_{m}\right) \in\{1, \ldots, n\}^{m}$, with $i_{1}<\cdots<i_{m}$ and $m \in\{1, \ldots, n\}$, we denote by $\boldsymbol{I}=\left(I_{1}, \ldots, I_{n-m}\right)$ its complement in $\{1, \ldots, n\}$. The definition of $D$ gives $Y_{1} \wedge \cdots \wedge Y_{n}=D \partial_{1} \wedge \cdots \wedge \partial_{n}$. If we take the interior product of this equation with $d x_{I}=d x_{I_{1}} \wedge \cdots \wedge d x_{I_{n-m}}$, we get

$$
D \partial_{i}=\sum_{k}(-1)^{|i|+|k|} L_{k i} Y_{k},
$$

where $\boldsymbol{k}$ is a subscript analogous to $\boldsymbol{i}$, where $\partial_{\boldsymbol{i}}$ and $Y_{\boldsymbol{k}}$ are compact notations similar to $d x_{I}$, where $|\cdot|$ is the sum of the components, and where $L_{k i}$ denotes some homogeneous polynomial. Setting $L^{k i}:=L_{K I}$, we have a theorem:

Theorem 10. (i) There is a canonical nonsurjective injection $i: \mathscr{R} \rightarrow \mathscr{P}$.

(ii) A homogeneous potential cochain $D^{-1} \sum_{k} P^{k r} Y_{k}$ (of bidegree $(p, r)$, where $p$ is the exterior degree and $r$ the polynomial degree) is real if and only if the $n ! / p !(n-p)$ ! homogeneous polynomials $\sum_{k} L^{k i} P^{k r}$ (of degree $\left.p+r\right)$ are divisible by $D$; in case $p=0$ this condition means that $P^{r}$ is divisible by $D$.

Remark. The bigrading $\mathscr{P}=\bigoplus_{p=0}^{n} \bigoplus_{r=0}^{\infty} \mathscr{P}^{p r}$, defined on $\mathscr{P}$ by the exterior degree and the polynomial degree, induces a bigrading $\mathscr{R}=\bigoplus_{p=0}^{n} \bigoplus_{r=0}^{\infty} \mathscr{R}^{p r}$ on $\mathscr{R}$.

Consider now a quadratic Poisson tensor $\Lambda$ in $\mathbb{R}^{n}$. From now on, we assume that $\Lambda$ is SRMI, and more precisely that there are $n$ mutually commuting linear vector fields $Y_{i}=\sum_{r=1}^{n} \ell_{i r} \partial_{r}$ with $\ell \in \mathfrak{g l}\left(n, \mathbb{R}^{n *}\right)$ such that $D=\operatorname{det} \ell \neq 0$ and

$$
\Lambda=\sum_{i<j} \alpha^{i j} Y_{i j}, \quad \text { where } \alpha^{i j} \in \mathbb{R} .
$$

Proposition 11. The determinant $D=\operatorname{det} \ell \in \mathscr{S}^{n} \mathbb{R}^{n *} \backslash\{0\}$ is the unique joint eigenvector of the $Y_{i}$ with eigenvalues $\operatorname{div} Y_{i} \in \mathbb{R}$, that is, $D$ is up to multiplication by nonzero constants the unique nonzero polynomial of $\mathbb{R}^{n}$ that satisfies

$$
Y_{i} D=\left(\operatorname{div} Y_{i}\right) D \quad \text { for all } i \in\{1, \ldots, n\} .
$$


Moreover, if $D=D_{1} D_{2}$, where $D_{1} \in \varphi^{n_{1}} \mathbb{R}^{n *}$ and $D_{2} \in \varphi^{n_{2}} \mathbb{R}^{n *}$ with $n_{1}+n_{2}=n$ are two polynomials without common divisor, these factors $D_{1}$ and $D_{2}$ are also joint eigenvectors. Their eigenvalues $\lambda_{i}$ and $\mu_{i}$ satisfy $\lambda_{i}+\mu_{i}=\operatorname{div} Y_{i}$.

Proof. For $i \in\{1, \ldots, n\}$,

$$
\begin{aligned}
0=\left[Y_{i}, Y_{1} \wedge \cdots \wedge Y_{n}\right] & =\left[Y_{i}, D \partial_{1} \wedge \cdots \wedge \partial_{n}\right] \\
& =\left(Y_{i} D\right) \partial_{1} \wedge \cdots \wedge \partial_{n}-D\left(\operatorname{div} Y_{i}\right) \partial_{1} \wedge \cdots \wedge \partial_{n},
\end{aligned}
$$

so that $Y_{i} D=\left(\operatorname{div} Y_{i}\right) D$.

For uniqueness, let $P \in \mathscr{S} \mathbb{R}^{n *} \backslash\{0\}$ be another polynomial such that $Y_{i} P=$ $\left(\operatorname{div} Y_{i}\right) P$ for all $i \in\{1, \ldots, n\}$. Then $Y_{i}(P / D)=0$ in $Z=\left\{x \in \mathbb{R}^{n}, D(x) \neq 0\right\}$, and, reasoning as in the proof of Theorem 1 , we conclude there exists $\alpha \in \mathbb{R}^{*}$ such that $P=\alpha D$.

Finally, because $\left(\left(\operatorname{div} Y_{i}\right) D_{1}-Y_{i} D_{1}\right) D_{2}=D_{1}\left(Y_{i} D_{2}\right)$ and the polynomials $D_{1}$ and $D_{2}$ have no common divisor, $Y_{i} D_{2}=P D_{2}$ and $\left(\operatorname{div} Y_{i}\right) D_{1}-Y_{i} D_{1}=Q D_{1}$, where $P=Q$ is a polynomial. Looking at degrees, we see $P=Q$ is constant.

Remark. The eigenvalues $\operatorname{div} Y_{i}$ for $i \in\{1, \ldots, n\}$ cannot vanish simultaneously, for otherwise the polynomial $D \in \mathscr{G}^{n} \mathbb{R}^{n *} \backslash\{0\}$ vanishes everywhere.

Definition 12. The complex $0 \rightarrow \mathscr{R}^{0} \rightarrow \mathscr{R}^{1} \rightarrow \cdots \rightarrow \mathscr{R}^{n} \rightarrow 0$ with differential $\partial_{\Lambda}=[\Lambda, \cdot]_{S N}$ is the formal LP-complex of Poisson tensor $\Lambda \in \mathscr{S}^{2} \mathbb{R}^{n *} \otimes \Lambda^{2} \mathbb{R}^{n}$. We denote the corresponding cohomology groups by $L H^{*}(\Re, \Lambda)$.

The next theorem shows that if the cochains $C \in \mathscr{R}$ are read as $C=i C \in \mathscr{P}$, the LP-differential simplifies.

Theorem 13. Set $\Lambda=\sum_{i<j} \alpha^{i j} Y_{i j}, \alpha^{j i}=-\alpha^{i j}$, and $X_{i}=\sum_{j \neq i} \alpha^{i j} Y_{j}$.

(i) Let $C=D^{-1} \sum_{k} P^{k r} Y_{k} \in \mathscr{P P}^{p r}$ be a homogeneous potential cochain. The LP-coboundary of $C$ is given by

$$
\begin{aligned}
\partial_{\Lambda} C & =\sum_{k i} X_{i}\left(D^{-1} P^{k r}\right) Y_{i} \wedge Y_{k} \\
& =D^{-1} \sum_{k i}\left(X_{i}-\delta_{i} \text { id }\right)\left(P^{k r}\right) Y_{i} \wedge Y_{\boldsymbol{k}} \in \mathscr{P}^{p+1, r},
\end{aligned}
$$

where $\delta_{i}=\operatorname{div} X_{i} \in \mathbb{R}$.

(ii) The LP-coboundary operator $\partial_{\Lambda}$ endows $\mathscr{P}$ with a differential complex structure and preserves the polynomial degree $r$. This LP-complex of $\Lambda$ over $\mathscr{P}$ contains the LP-complex $\left(\Re, \partial_{\Lambda}\right)$ of $\Lambda$ over $\Re$ as a differential subcomplex.

Proof. If $C=f \boldsymbol{Y}$, with $f$ a function and $\boldsymbol{Y}$ a wedge product of vector fields $Y_{k}$, we get

$$
\partial_{\Lambda}(f \boldsymbol{Y})=[\Lambda, f \boldsymbol{Y}]_{\mathrm{SN}}=[\Lambda, f]_{\mathrm{SN}} \wedge \boldsymbol{Y}
$$


since the $Y_{k}$ commute. However,

$$
\begin{aligned}
{[\Lambda, f]_{\mathrm{SN}} } & =\sum_{i<j} \alpha^{i j}\left(\left(Y_{j} f\right) Y_{i}-\left(Y_{i} f\right) Y_{j}\right) \\
& =\sum_{i}\left(\sum_{j \neq i} \alpha^{i j} Y_{j} f\right) Y_{i}=\sum_{i}\left(X_{i} f\right) Y_{i} .
\end{aligned}
$$

By combining (3-5) and (3-6), we get the first part of (3-4), whereas its second part is a consequence of Proposition 11.

Corollary 14. The LP-cohomology groups of $\Lambda$ over $\mathscr{R}$ and $\mathscr{P}$ are bigraded, that is,

$$
L H(\mathscr{R}, \Lambda)=\bigoplus_{r=0}^{\infty} \bigoplus_{p=0}^{n} L H^{p r}(\mathscr{R}, \Lambda) \quad \text { and } \quad L H(\mathscr{P}, \Lambda)=\bigoplus_{r=0}^{\infty} \bigoplus_{p=0}^{n} L H^{p r}(\mathscr{P}, \Lambda),
$$

where for instance $L H^{p r}(\mathscr{P}, \Lambda)$ is defined by

$$
L H^{p r}(\mathscr{P}, \Lambda)=\operatorname{ker}\left(\partial_{\Lambda}: \mathscr{P}^{p r} \rightarrow \mathscr{P}^{p+1, r}\right) / \operatorname{im}\left(\partial_{\Lambda}: \mathscr{P}^{p-1, r} \rightarrow \mathscr{P}^{p r}\right) .
$$

In the following we deal with the terms $L P^{* r}(\mathscr{P}, \Lambda)=\bigoplus_{p=0}^{n} L P^{p r}(\mathscr{P}, \Lambda)$ of the LP-cohomology over $\mathscr{P}$ and with the corresponding part of the LP-cohomology of the subcomplex $\mathscr{R}$.

Theorem 15. Let $E_{r}$ be the real finite-dimensional vector space $\varphi^{r} \mathbb{R}^{n *}$, and let $\vec{X}_{\delta}:=\left(X_{1}-\delta_{1} \mathrm{id}, \ldots, X_{n}-\delta_{n} \mathrm{id}\right)$, where $\delta_{i}=\operatorname{div} X_{i}$, be the $n$-tuple of commuting linear operators $X_{i}-\delta_{i}$ id on $E_{r}$ defined in Theorem 13. The LP-cohomology space $L H^{* r}(\mathscr{P}, \Lambda)$ coincides with the Koszul cohomology space $K H^{*}\left(\vec{X}_{\delta}, E_{r}\right)$.

Proof. This follows from $\partial_{\Lambda}=\sum_{i}\left(X_{i}-\delta_{i}\right.$ id $) \otimes e_{Y_{i}}$, as proved in Theorem 13.

Since $\left(\mathscr{R}, \partial_{\Lambda}\right)$ is a subcomplex of $\left(\mathscr{P}, \partial_{\Lambda}\right)$, we can use classical techniques (namely, the long exact cohomology sequence) to deduce the LP-cohomology of $\Lambda$ from the Koszul cohomology associated with $\vec{X}_{\delta}$. More precisely, consider the relative cohomology $L H(\mathscr{P}, \mathscr{R}, \Lambda)$ of $\left(\mathscr{P}, \partial_{\Lambda}\right)$ with respect to $\mathscr{R}$, that is, the cohomology of the space $\left(\mathscr{P} / \mathscr{R}, \bar{\partial}_{\Lambda}\right)$, and let $\phi$ be the composition of $\partial_{\Lambda}$ with the projection of $\mathscr{P}$ onto $\mathscr{R}$.

Theorem 16. The LP-cohomology groups of a SRMI Poisson tensor $\Lambda$ over the space $\mathscr{R}$ of cochains with coefficients in formal power series are given by

$$
L H^{p r}(\mathscr{R}, \Lambda) \simeq L H^{p r}(\mathscr{P}, \Lambda) / \operatorname{ker}^{p r} \phi_{\sharp} \oplus L H^{p-1, r}(\mathscr{P}, \mathscr{R}, \Lambda) / \operatorname{ker}^{p-1, r} \phi_{\sharp} .
$$

Remark 1. This theorem reduces computing the groups $L H^{p r}(\mathscr{R}, \Lambda)$ to finding the groups $L H^{p r}(\mathscr{P}, \Lambda) \simeq K H^{p}\left(\vec{X}_{\delta}, E_{r}\right)$ associated to the operators $\vec{X}_{\delta}$ on $E_{r}=\mathscr{S}^{r} \mathbb{R}^{n *}$ induced by $\Lambda$, and to finding the relative cohomology groups $L H^{p-1, r}(\mathscr{P}, \mathscr{R}, \Lambda)$. It thus links Poisson and Koszul cohomology. In [Masmoudi and Poncin 2007], 
we showed via explicit computations in $\mathbb{R}^{3}$ that $\mathscr{P}$-cohomology (now identified as Koszul cohomology) and $\mathscr{Y}$-cohomology (or relative cohomology) are less intricate than Poisson cohomology.

\section{Koszul cohomology in a finite-dimensional vector space}

In view of Remark 1, we now turn to the Koszul cohomology space $K H^{*}\left(\vec{X}_{\lambda}, E\right)$ associated to operators $\vec{X}_{\lambda}:=\left(X_{1}-\lambda_{1}\right.$ id, $\ldots, X_{n}-\lambda_{n}$ id $)$ made up of commuting linear transformations $\vec{X}:=\left(X_{1}, \ldots, X_{n}\right)$ of a finite-dimensional real vector space $E$ and a point $\vec{\lambda}:=\left(\lambda_{1}, \ldots, \lambda_{n}\right) \in \mathbb{R}^{n}$. Koszul cohomology is known to be closely connected with spectral theory: A fundamental principle of multivariate operator theory is that all essential spectral properties of operators $\vec{X}$ in a complex space should be understood in terms of properties of the Koszul complex induced by $\vec{X}_{\lambda}$ for $\vec{\lambda} \in \mathbb{C}^{n}$. Thus the complex setting is the natural one for investigating Koszul cohomology. To engage this point of view, it suffices to note that, if $\vec{X} \in \operatorname{End}_{\mathbb{R}}(E)$ are commuting $\mathbb{R}$-linear transformations of a real vector space $E$, and if $\vec{X}^{\mathbb{C}} \in \operatorname{End}_{\mathbb{C}}\left(E^{\mathbb{C}}\right)$ are the corresponding commuting complexified $\mathbb{C}$-linear transformations of the complexification $E^{\mathbb{C}}$ of $E$, the cohomology $K H^{*}\left(\vec{X}^{\mathbb{C}}, E^{\mathbb{C}}\right)$ of the complexification $K^{*}\left(\vec{X}^{\mathbb{C}}, E^{\mathbb{C}}\right)$ of the complex $K^{*}(\vec{X}, E)$ is isomorphic to the complexification $K H^{* \mathbb{C}}(\vec{X}, E)$ of the cohomology of $K^{*}(\vec{X}, E)$.

Below, we use the concept of joint spectrum $\sigma(\vec{X})$ of commuting bounded linear operators $\vec{X}=\left(X_{1}, \ldots, X_{n}\right)$ on a complex vector space $E$. Such spectra are defined variously in the literature, where $E$ may be a normed space, a Banach space, or a Hilbert space. Here we investigate Koszul cohomology in finite dimension and need the following characterizations of the elements of the joint spectrum $\sigma(\vec{X})$; for a proof, see [Bolotnikov and Rodman 2002].

Proposition 17. Let $\vec{X}=\left(X_{1}, \ldots, X_{n}\right)$ be an $n$-tuple of commuting operators on a finite-dimensional complex vector space $E$. Then these statements are equivalent for any fixed $\vec{\lambda}=\left(\lambda_{1}, \ldots, \lambda_{n}\right) \in \mathbb{C}^{n}$ :

(a) $\vec{\lambda} \in \sigma(\vec{X})$.

(b) There is a basis in $E$ in which the matrices representing the $X_{j}$ are all uppertriangular, and there is an index $q$ in $1 \leq q \leq \operatorname{dim} E$ such that $\lambda_{j}$ is the $(q, q)$ entry of the matrix representing $X_{j}$ for $j \in\{1, \ldots, n\}$.

(c) For every basis in $E$ in which matrices for the $X_{j}$ are all upper-triangular, there is an index $q$ as in (b).

(d) There is a nonzero vector $x$ such that $X_{j} x=\lambda_{j} x$ for all $j \in\{1, \ldots, n\}$

(e) There are no $Y_{j}$ in the subalgebra of $\operatorname{End}_{\mathbb{C}}(E)$ generated by id and $\vec{X}$ that satisfy $\sum_{j=1}^{n} Y_{j}\left(X_{j}-\lambda_{j} \mathrm{id}\right)=\mathrm{id}$. 
We now supply some results about Koszul cohomology spaces, using the same notation as above. The first is obvious.

Proposition 18. Let $\bigwedge=\bigwedge_{n}\langle\vec{\eta}\rangle$ be the exterior algebra on $n$ with generators $\vec{\eta}$ over a field $\mathbb{F}$ of characteristic 0 , and let $\vec{h}$ be the dual generators, that is, suppose $i_{h_{k}} \eta_{\ell}=\partial_{k \ell}$. We then have the homotopy formula $e_{\eta_{\ell}} i_{h_{k}}+i_{h_{k}} e_{\eta_{\ell}}=\delta_{k \ell}$ id, where $i_{h_{k}}$ and $e_{\eta_{\ell}}$ are the creation and annihilation operators, respectively.

Proposition 19. Let $\overrightarrow{\mathscr{X}} \in \operatorname{End}_{\mathbb{F}}^{\times n}(E)$ and $\vec{Y} \in \operatorname{End}_{\mathbb{F}}^{\times n}(E)$ be $n$ commuting linear operators $\overrightarrow{\mathscr{X}}$ and $\vec{Y}$, respectively, on a vector space $E$ over $\mathbb{F}$. We denote by $\mathscr{K}=$ $\sum_{\ell} \mathscr{X}_{\ell} \otimes e_{\eta_{\ell}}$ and $\kappa=\sum_{k} Y_{k} \otimes i_{h_{k}}$ the respective corresponding Koszul cohomology and homology operators. Then

$$
\mathscr{K} \kappa+\kappa \mathscr{K}=\left(\sum_{\ell} Y_{\ell} \mathscr{X}_{\ell}\right) \otimes \mathrm{id}+\sum_{k \ell}\left[\mathscr{X}_{\ell}, Y_{k}\right] \otimes e_{\eta_{\ell}} i_{h_{k}} .
$$

Proof. This is a direct consequence of Proposition 18.

Theorem 20. Let $\vec{X} \in \operatorname{End}_{\mathbb{C}}^{\times n}(E)$ be $n$ commuting endomorphisms of E, a finitedimensional complex vector space, and let $\vec{\lambda} \in \mathbb{C}^{n}$. For splitting $E=E_{1} \oplus E_{2}$, denote by $i_{j}: E_{j} \rightarrow E$ the injection of $E_{j}$ into $E$ and by $p_{j}: E \rightarrow E_{j}$ the projection of $E$ onto $E_{j}$.

If $E_{1}$ is stable under the operators $X_{\ell}$, that is, $p_{2} X_{\ell} i_{1}=0$, and if $\vec{\lambda}$ is not in the joint spectrum $\sigma\left(\vec{X}^{\prime}\right)$ of the commuting operators $X_{\ell}^{\prime}=p_{2} X_{\ell} i_{2} \in \operatorname{End}_{\mathbb{C}}\left(E_{2}\right)$, then any cocycle $C \in E \otimes \bigwedge$ of the Koszul complex $K^{*}\left(\vec{X}_{\lambda}, E\right)$, where $\vec{X}_{\lambda}=\vec{X}-\vec{\lambda} \mathrm{id}_{E}$, is cohomologous to a cocycle $C_{1} \in E_{1} \otimes \bigwedge$, with $\bigwedge=\bigwedge_{n}\langle\vec{\eta}\rangle$.

Proof. If $q(\vec{X}) \in \mathbb{C}\left[X_{1}, \ldots, X_{n}\right] \subset \operatorname{End}_{\mathbb{C}}(E)$ denotes a polynomial in the $X_{\ell}$, the map $q(\vec{X})_{2}=p_{2} q(\vec{X}) i_{2}$ coincides with the (same) polynomial $q\left(\vec{X}^{\prime}\right) \in \operatorname{End}_{\mathbb{C}}\left(E_{2}\right)$ in the $X_{\ell}^{\prime}$. Indeed, due to stability of $E_{1}$, we have

$$
p_{2} X_{\ell} X_{k} i_{2}=p_{2} X_{\ell} i_{1} p_{1} X_{k} i_{2}+p_{2} X_{\ell} i_{2} p_{2} X_{k} i_{2}=X_{\ell}^{\prime} X_{2}^{\prime}
$$

This implies that the $X_{\ell}^{\prime}$ commute.

Since $\vec{\lambda} \notin \sigma\left(\vec{X}^{\prime}\right)$, Proposition 17(e) implies that there are $n$ operators $\vec{Y}^{\prime}$ in the subalgebra of $\operatorname{End}_{\mathbb{C}}\left(E_{2}\right)$ generated by $\operatorname{id}_{E_{2}}$ and $\vec{X}^{\prime}$ such that

$$
\sum_{\ell} Y_{\ell}^{\prime}\left(X_{\ell}^{\prime}-\lambda_{\ell} \mathrm{id}_{E_{2}}\right)=\mathrm{id}_{E_{2}}
$$

Hence $Y_{\ell}^{\prime}=Q_{\ell}\left(\vec{X}^{\prime}\right)$ is a polynomial in the $X_{k}^{\prime}$ for any $\ell$. Set $Y_{\ell}=Q_{\ell}(\vec{X}) \in \operatorname{End}_{\mathbb{C}}(E)$. If applied to operators $\vec{X}_{\lambda}$ and $\vec{Y}$, Proposition 19 implies that

$$
\left(\sum_{\ell} Y_{\ell}\left(X_{\ell}-\lambda_{\ell} \mathrm{id}_{E}\right)\right) \otimes \mathrm{id}_{\wedge}+\sum_{k \ell}\left[X_{\ell}-\lambda_{\ell} \mathrm{id}_{E}, Y_{k}\right] \otimes e_{\eta_{\ell}} i_{h_{k}}=\mathscr{K} \kappa+\kappa \mathscr{K},
$$


where $\mathscr{K}$ and $\kappa$ are respectively the Koszul cohomology and homology operators associated with $\vec{X}_{\lambda}$ and $\vec{Y}$ on $E$. Since $Y_{k}$ is a polynomial in the commuting endomorphisms $X_{\ell}$, the second term on the left side of (4-2) vanishes. Hence, when evaluating both sides on a cocycle $C=e \otimes w$ of cochain complex $K^{*}\left(\vec{X}_{\lambda}, E\right)$, we get

$$
(Q(\vec{X})(e)) w=\mathscr{K} \kappa(e \otimes w),
$$

where $Q(\vec{X})=\sum_{\ell} Y_{\ell}\left(X_{\ell}-\lambda_{\ell} \operatorname{id}_{E}\right)=\sum_{\ell} Q_{\ell}(\vec{X})\left(X_{\ell}-\lambda_{\ell} \operatorname{id}_{E}\right)$ is a polynomial in the $X_{\ell}$. Absent the factor $w$, the left side reads

$$
Q(\vec{X})(e)=p_{1} Q(\vec{X}) i_{1} p_{1}(e)+p_{2} Q(\vec{X}) i_{1} p_{1}(e)+p_{1} Q(\vec{X}) i_{2} p_{2}(e)+p_{2} Q(\vec{X}) i_{2} p_{2}(e),
$$

where the second term on the right vanishes in view of the stability of $E_{1}$, and the last term equals $p_{2}(e)$, in view of the first sentence of the proof of Theorem 20 and (4-1). Finally, the cocycle $C=e \otimes w$ is cohomologous to

$$
C_{1}=C-\mathscr{T} \kappa C=\left(p_{1}(e)-p_{1} Q(\vec{X}) i_{1} p_{1}(e)-p_{1} Q(\vec{X}) i_{2} p_{2}(e)\right) \otimes w \in E_{1} \otimes \bigwedge .
$$

This theorem has a number of new and partially practical consequences.

First, if $\vec{X} \in \operatorname{End}_{\mathbb{C}}^{\times n}(E)$ are $n$ commuting $\mathbb{C}$-linear endomorphisms, the complex finite-dimensional vector space $E$ on which these operators act has a direct sum decomposition $E=\bigoplus_{\vec{\mu} \in \mathbb{C}^{n}} E^{\mu}$, where the primary subspace of $E$ associated with the weight $\vec{\mu}$, namely

$$
E^{\mu}=\bigcap_{i} E^{\mu_{i}}=\bigcap_{i} \bigcup_{n \in \mathbb{N}} \operatorname{ker}\left(X_{i}-\mu_{i} \mathrm{id}\right)^{n}
$$

is stable under the action of the operators $\vec{X}$ [Bourbaki 1975, théorème 1]. Let us also mention that

$$
E^{\mu_{i}}=\bigcup_{n \in \mathbb{N}} \operatorname{ker}\left(X_{i}-\mu_{i} \mathrm{id}\right)^{n}=\operatorname{ker}\left(X_{i}-\mu_{i} \mathrm{id}\right)^{m_{i}},
$$

where $m_{i}$ denotes the multiplicity of the solution $\mu_{i}$ of the characteristic polynomial of $X_{i}$, and that $\operatorname{dim} E^{\mu_{i}}=m_{i}$. Since the multiplicity $\mathfrak{m}$ of $\overrightarrow{0}$ in the joint spectrum of the commuting operators $X_{i}-\mu_{i}$ id coincides with its multiplicity in the joint spectrum of the operators $\left(X_{i}-\mu_{i} \text { id }\right)^{m}$, where $m=\sup \left\{m_{i}\right\}$, we easily see that the dimension of $E^{\mu}=\bigcap_{i} \operatorname{ker}\left(X_{i}-\mu_{i} \text { id }\right)^{m}$ cannot exceed $\mathfrak{m}$.

Another consequence of Theorem 20 is that Koszul cohomology $K H^{*}\left(\vec{X}_{\lambda}, E\right)$, roughly speaking, is made up of weak joint eigenvectors with eigenvalues $\lambda_{\ell}$.

Corollary 21. Let $\vec{\lambda} \in \mathbb{C}^{n}$, and let $\vec{X} \in \operatorname{End}_{\mathbb{C}}^{\times n}(E)$ be $n$ commuting endomorphisms of a finite-dimensional complex vector space E. Denote by $\Lambda=\bigwedge_{n}\langle\vec{\eta}\rangle$ the Grassmann algebra with $n$ generators $\vec{\eta}$. Any cocycle $C \in E \otimes \wedge$ of the Koszul complex $K^{*}\left(\vec{X}_{\lambda}, E\right)$ is cohomologous to a cocycle $C_{1} \in E^{\lambda} \otimes \bigwedge$. 
This corollary has a useful variant. Choose a supplementary subspace $E^{(2)}$ in $E$ of the stable subspace $\operatorname{ker} \vec{X}_{\lambda}:=\bigcap_{\ell} \operatorname{ker}\left(X_{\ell}-\lambda_{\ell}\right.$ id $)$ of joint eigenvectors, and denote by $\vec{X}^{(2)}$ the restrictions of the operators $\vec{X}$ to $E^{(2)}$; see Theorem 20 . We can iterate this procedure, choosing a supplementary subspace $E^{(3)}$ in $E^{(2)}$ of $\operatorname{ker} \vec{X}_{\lambda}^{(2)}$, introducing the restrictions $\vec{X}^{(3)}$ of the operators $\vec{X}^{(2)}$ to $E^{(3)}$, and so on, until we get $\operatorname{ker} \vec{X}_{\lambda}^{(s+1)}=0$. We will show that the direct sum of these kernels coincides with the space $E^{\lambda}$ above, so that Corollary 21 means that any cocycle $C \in E \otimes \wedge$ of $K^{*}\left(\vec{X}_{\lambda}, E\right)$ is cohomologous to a cocycle $C_{1} \in \bigoplus_{a=1}^{s} \operatorname{ker} \vec{X}_{\lambda}^{(a)} \otimes \bigwedge$, where of course $\operatorname{ker} \vec{X}_{\lambda}^{(1)}=\operatorname{ker} \vec{X}_{\lambda}$.

Proof of Corollary 21. Let $E_{\lambda}:=\operatorname{ker} \vec{X}_{\lambda} \subset E^{\lambda}$ and denote by $E(\lambda)$ a supplementary subspace of $E_{\lambda}$ in $E^{\lambda}$. Set

$$
E=E^{\lambda} \oplus \bigoplus_{\vec{\mu} \neq \vec{\lambda}} E^{\mu}=: E_{1} \oplus E_{2}
$$

and use the notation of Theorem 20. Assume that $\vec{\lambda} \in \sigma\left(\vec{X}^{\prime}\right)$, that is, that $\operatorname{ker} \vec{X}_{\lambda}^{\prime} \neq 0$. If $e \in E_{2}$ is a nonvanishing joint eigenvector of the $X_{\ell}^{\prime}=p_{2} X_{\ell} i_{2}$ with eigenvalues $\lambda_{\ell}$, we have $X_{\ell} e=p_{1} X_{\ell} e+\lambda_{\ell} e$ for any $\ell$. Since $E_{2}$ is fixed by the $X_{\ell}$, we get $p_{1} X_{\ell} e=0$, so that $e \in E_{1} \cap E_{2}=0$, a contradiction; finally $\vec{\lambda} \notin \sigma\left(\vec{X}^{\prime}\right)$ and we finish using Theorem 20.

On the other hand, set

$$
E=E_{\lambda} \oplus\left(E(\lambda) \oplus \bigoplus_{\vec{\mu} \neq \vec{\lambda}} E^{\mu}\right)=: E_{1} \oplus E_{2} .
$$

Note that the operators $\vec{X}^{\prime}$ in this decomposition coincide with the $\vec{X}^{(2)}$ above. Now $\left(X_{\ell}-\lambda_{\ell} \mathrm{id}\right) e=p_{1} X_{\ell} e \in E_{1}=\operatorname{ker} \vec{X}_{\lambda}$. Any $e \in \operatorname{ker} \vec{X}_{\lambda}^{\prime}=\operatorname{ker} \vec{X}_{\lambda}^{(2)}$ belongs to $E^{\lambda}$. Also ker $\vec{X}_{\lambda} \oplus \operatorname{ker} \vec{X}_{\lambda}^{(2)} \subset E^{\lambda}$; more generally, $\bigoplus_{a=1}^{s} \operatorname{ker} \vec{X}_{\lambda}^{(a)} \subset E^{\lambda}$. Since, as is easily checked, the dimension of this direct sum is equal to the multiplicity $\mathfrak{m}$ (which is no less than $\operatorname{dim} E^{\lambda}$ ) of $\overrightarrow{0}$ in the joint spectrum $\sigma\left(\vec{X}_{\lambda}\right)$, this direct sum coincides with $E^{\lambda}$.

We next recover a well-known result, and then an important special case.

Corollary 22. Let $\vec{X} \in \operatorname{End}_{\mathbb{C}}^{\times n}(E)$ be $n$ commuting endomorphisms, and let $\vec{\lambda} \in \mathbb{C}^{n}$. Then $K H^{*}\left(\vec{X}_{\lambda}, E\right)$ is trivial if and only if $\operatorname{dim}\left(\operatorname{ker} \vec{X}_{\lambda}\right)=0$.

Corollary 23. Assume the conditions of Corollary 21. If for any $\ell \in\{1, \ldots, n\}$ the kernel and image of $X_{\ell}-\lambda_{\ell}$ id are supplementary in $E$, then any cocycle $C \in E \otimes \wedge$ of the Koszul complex $K^{*}\left(\vec{X}_{\lambda}, E\right)$ is cohomologous to a cocycle $C_{1} \in \operatorname{ker} \vec{X}_{\lambda} \otimes \bigwedge$. Proofs. First the forward implication in Corollary 22. If there exists $e \in \operatorname{ker} \vec{X}_{\lambda} \backslash\{0\}$, then

$$
\mathscr{K}_{\vec{X}_{\lambda}} e=\sum_{\ell=1}^{n}\left(X_{\ell}-\lambda_{\ell} \mathrm{id}\right) e \eta_{\ell}=0,
$$


so that $e$ is a nonbounding 0 -cocycle. As for Corollary 23, it follows from the proof of Corollary 21 that if there is a nonzero vector $e \in \operatorname{ker} \vec{X}_{\lambda}^{(2)} \subset E^{(2)}$, then for every $\ell$ we have $\left(X_{\ell}-\lambda_{\ell}\right.$ id $) e \in \operatorname{ker} \vec{X}_{\lambda} \cap \operatorname{im}\left(X_{\ell}-\lambda_{\ell}\right.$ id $)=0$, so that $e \in \operatorname{ker} \vec{X}_{\lambda} \cap E^{(2)}=0$, which is a contradiction.

\section{Koszul cohomology associated with Poisson cohomology}

We now return to the Koszul cohomology implemented by a SRMI tensor of $\mathbb{R}^{n}$. Recall the QPT tensor $\Lambda$ from (1-1) and the conditions under which it is SRMI. Theorems 13 and 15 identify the main building block of the LP-cohomology of $\Lambda$ as the Koszul cohomology space $K H^{*}\left(\vec{X}_{\delta}, E_{r}\right)$. We noted that this cohomology can be deduced from its complex counterpart $K H^{*}\left(\vec{X}_{\delta}^{\mathbb{C}}, E_{r}^{\mathbb{C}}\right)$, which, according to Corollaries 21-23, is closely related to the joint eigenvectors and spectrum of $\vec{X}^{\mathbb{C}}$ or $\vec{X}_{\delta}^{\mathbb{C}}$. We now further investigate $K H^{*}\left(\vec{X}_{\delta}^{\mathbb{C}}, E_{r}^{\mathbb{C}}\right)$. In particular, we reduce its computation to a problem of linear algebra, and describe the spectrum of the commuting transformations $\vec{X}_{\delta}^{\mathbb{C}}$.

Proposition 24. Let $a_{j}=J^{-1} Y_{j} \in \mathfrak{g l}(n, \mathbb{R})$ for $j \in\{1, \ldots, n\}$. Any basis of $\mathbb{C}^{n}$ in which the $\vec{a}$ are upper-triangular naturally induces a basis of $E_{r}^{\mathbb{C}}=\varphi^{r} \mathbb{C}^{n *}$ in which the $\vec{X}_{\delta}^{\complement}$ are upper-triangular.

In what follows, the use of super- and subscripts is dictated by aesthetics and not by contra- or covariance.

Proof. Let $x=\left(x_{1}, \ldots, x_{n}\right) \in \mathbb{R}^{n}$ and $z=\left(z_{1}, \ldots, z_{n}\right) \in C^{n}$. As usual, we set $Y_{k}=\sum_{m} \ell_{k m} \partial_{x_{m}}=\sum_{m p} a_{k}^{m p} x_{p} \partial_{x_{m}}$ and use notations as $x^{\beta}=x_{1}^{\beta_{1}} \cdots x_{n}^{\beta_{n}}$ for $\beta \in \mathbb{N}^{n}$.

We complexify

$$
E_{r}=\mathscr{Y}^{r} \mathbb{R}^{n *}=\left\{P \in C^{\infty}\left(\mathbb{R}^{n}\right): P(x)=\sum_{|\beta|=r} r_{\beta} x^{\beta}, x \in \mathbb{R}^{n}, r_{\beta} \in \mathbb{R}\right\}
$$

to $E_{r}^{\mathbb{C}} \simeq \mathscr{Y}^{r} \mathbb{C}^{n *}$ by replacing $\mathbb{R}$ with $\mathbb{C}$. The complexification $Y_{k}^{\mathbb{C}} \in \operatorname{End}_{\mathbb{C}}\left(E_{r}^{\mathbb{C}}\right)$ of $Y_{k} \in \operatorname{End}_{\mathbb{R}}\left(E_{r}\right)$ is the holomorphic vector field

$$
Y_{k}^{\mathbb{C}}=\sum_{m p} a_{k}^{m p} z_{p} \partial_{z_{m}} \in \operatorname{Vect}^{10}\left(\mathbb{C}^{n}\right) \quad \text { of } \mathbb{C}^{n} .
$$

There is a unitary matrix $U \in \mathrm{U}(n, \mathbb{C})$ such that $b_{j}=U^{-1} a_{j} U$ is upper-triangular, and there is a corresponding basis $\left(e_{1}^{\prime}, \ldots, e_{n}^{\prime}\right)$ of $\mathbb{C}^{n}$ such that the $a_{j}$ themselves are upper-triangular. Let $\left(\varepsilon_{1}^{\prime}, \ldots, \varepsilon_{n}^{\prime}\right)$ be the corresponding dual basis.

Express $z$ in this basis as $z=\sum_{j} \mathfrak{z}_{j} e_{j}^{\prime} \in \mathbb{C}^{n}$. If viewed as a basis of the space $E_{r}^{\mathbb{C}}$ of degree $r$ homogeneous polynomials of $\mathbb{C}^{n}$, the induced basis of the space $\varphi^{r} \mathbb{C}^{n *}$ of symmetric covariant $r$-tensors of $\mathbb{C}^{n}$ is the set $\mathfrak{z}^{\beta}$ with $\beta \in \mathbb{N}^{n}$ and $|\beta|=r$.

To find the matrices of the operators $\vec{X}_{\delta}^{\mathbb{C}}$ in the basis $\mathfrak{z}^{\beta}$, we arrange its elements by the lexicographic order $\prec$ and perform the coordinate change $z=U \mathfrak{z}$ and put 
$\partial_{z}={\widetilde{\partial_{\mathfrak{z}}}}^{-1} \partial_{\mathfrak{z}}$ in the first order differential operators $\left(X_{j}-\delta_{j} \text { id }\right)^{\mathbb{C}}$. We get

$$
\begin{aligned}
\left(X_{j}-\delta_{j} \mathrm{id}\right)^{\mathbb{C}} & =\sum_{k} \alpha^{j k} \sum_{m \leq p} b_{k}^{m p} \mathfrak{z}_{p} \partial_{\mathfrak{z} m}-\delta_{j} \mathrm{id}^{\mathbb{C}} \\
& =\sum_{k m} \alpha^{j k} b_{k}^{m m}\left(\mathfrak{z} m \partial_{\mathfrak{z} m}-\mathrm{id}^{\mathbb{C}}\right)+\sum_{k} \sum_{m<p} \alpha^{j k} b_{k}^{m p} \mathfrak{z} p \partial_{\mathfrak{z} m},
\end{aligned}
$$

since $\delta_{j}=\operatorname{div} X_{j}=\sum_{k m} \alpha^{j k} a_{k}^{m m}=\sum_{k m} \alpha^{j k} b_{k}^{m m}$. These operators are then uppertriangular in the $\mathfrak{z}^{\beta}$ basis, since

$$
\left(X_{j}-\delta_{j} \mathrm{id}\right)^{\mathbb{C}} \mathfrak{z}^{\beta}=\sum_{k m} \alpha^{j k} b_{k}^{m m}\left(\beta_{m}-1\right) \mathfrak{z}^{\beta}+\sum_{k} \sum_{m<p} \alpha^{j k} b_{k}^{m p} \beta_{m} \mathfrak{z}^{\beta-e_{m}+e_{p}},
$$

where $\mathfrak{z}^{\beta-e_{m}+e_{p}} \prec \mathfrak{z}^{\beta}$.

With matrices $b_{j}$ as above, let $B \in \mathfrak{g l}(n, \mathbb{C})$ be the matrix $B_{j k}=b_{j}^{k k}$.

Theorem 25. The joint spectrum $\sigma_{r}\left(\vec{X}_{\delta}^{\mathbb{C}}\right)$ of the $\vec{X}_{\delta}^{\mathbb{C}}$ is given by

$$
\sigma_{r}\left(\vec{X}_{\delta}^{\mathbb{C}}\right)=\left\{\alpha B I: I \in(\mathbb{N} \cup\{-1\})^{n},|I|=r-n\right\} \subset \mathbb{C}^{n}, \quad \text { where }|I|=\sum_{j} I_{j} .
$$

Proof. This follows directly from Proposition 17 and (5-1).

Remark. In Proposition 11, we showed $Y_{k} D=\left(\operatorname{div} Y_{k}\right) D$ that for all $k$, where $D=\operatorname{det} \ell \in E_{n} \subset E_{n}^{\mathbb{C}}$. Then $X_{j}^{\mathbb{C}} D=X_{j} D=\left(\operatorname{div} X_{j}\right) D=\delta_{j} \mathrm{id}^{\mathbb{C}} D$ for all $j$, so that $\overrightarrow{0}=(0, \ldots, 0) \in \sigma_{n}\left(\vec{X}_{\delta}^{\mathbb{C}}\right)$. This is immediately recovered from Theorem 25 .

Set $K_{r}\left(\vec{X}_{\delta}^{\mathbb{C}}\right)=\left\{I \in \operatorname{ker}(\alpha B): I \in(\mathbb{N} \cup\{-1\})^{n},|I|=r-n\right\}$. Corollary 22 can then be reformulated as follows.

Corollary 26. $K H^{*}\left(\vec{X}_{\delta}^{\complement}, E_{r}^{\mathbb{C}}\right)$ is acyclic if and only if $K_{r}\left(\vec{X}_{\delta}^{\mathbb{C}}\right)=\varnothing$.

Proof. $K H^{*}\left(\vec{X}_{\delta}^{\mathbb{C}}, E_{r}^{\mathbb{C}}\right)$ is trivial if and only if $\operatorname{dim}\left(\operatorname{ker} \vec{X}_{\delta}^{\mathbb{C}}\right)=0$, which is true if and only if $\overrightarrow{0} \notin \sigma_{r}\left(\vec{X}_{\delta}^{\mathbb{C}}\right)$, that is, if and only if $K_{r}\left(\vec{X}_{\delta}^{\mathbb{C}}\right)=\varnothing$.

Example 27. Consider the structure $\Lambda_{2}$ of the Dufour-Haraki classification as in Theorem 4 , and assume that $a \neq 0$ and $b=0$. It is easily checked that the matrix

$$
U=\left(\begin{array}{ccc}
0 & i / \sqrt{2} & -i / \sqrt{2} \\
0 & 1 / \sqrt{2} & 1 / \sqrt{2} \\
1 & 0 & 0
\end{array}\right)
$$

transforms the matrices $a_{\ell}$ into upper-triangular matrices $b_{\ell}$. A short computation shows that $K_{3 t}\left(\vec{X}_{\delta}^{\mathbb{C}}\right)$ for $t \in \mathbb{N}$ contains a single point $I_{t}=(t-1, t-1, t-1)$, so that the multiplicity $\mu$ of $\overrightarrow{0}$ in the joint spectrum $\sigma_{3 t}\left(\vec{X}_{\delta}^{\mathbb{C}}\right)$ equals 1 ; see the proof of Theorem 25. It follows that the $K H^{*}\left(\vec{X}_{\delta}^{\mathbb{C}}, E_{3 t}^{\mathbb{C}}\right)$ are not trivial; see Corollary 26. Furthermore, since the matrices $b_{\ell}$ are in fact diagonal in this case, (5-1) implies that $\mathfrak{z}_{1}^{t} \mathfrak{z}_{2}^{t} \mathfrak{z}_{3}^{t}$ belongs to the kernel of the $\vec{X}_{\delta}^{\mathbb{C}}$ in $E_{3 t}^{\mathbb{C}}$. Looking at dimensions, we see 
that this kernel is $\mathbb{C}_{\mathfrak{z}} \mathfrak{z}_{2}^{t} \mathfrak{z}_{2}^{t} \mathfrak{z}_{3}^{t}$ and that the reduced operators $\vec{X}_{\delta}^{\mathbb{C}(j)}$ for $j \in\{2, \ldots, s\}$ do not exist, that is, that $s=1$. Then, since the change to canonical coordinates is $z=U \mathfrak{z}$ by the proof of Proposition 24 , the space $K H^{p}\left(\vec{X}_{\delta}^{\mathbb{C}}, E_{3 t}^{\mathbb{C}}\right)$ for $p \in\{0,1,2,3\}$ and $t \in \mathbb{N}$ is contained in

$$
\mathfrak{z}_{1}^{t} \mathfrak{z}_{2}^{t} \mathfrak{z}_{3}^{t} \bigoplus_{j_{1}<\ldots<j_{p}} \mathbb{C} Y_{j_{1} \ldots j_{p}}=\left(z_{1}^{2}+z_{2}^{2}\right)^{t} z_{3}^{t} \bigoplus_{j_{1}<\ldots<j_{p}} \mathbb{C} Y_{j_{1} \ldots j_{p}}
$$

This easy consequence agrees with the results of [Masmoudi and Poncin 2007] modulo slight changes in notation - showing that the new approach is more efficient, though the same results could also be obtained via complexification.

Example 28. For $\Lambda_{3}$ of the Dufour-Haraki classification with parameter $a=0$, the multiplicity of $\overrightarrow{0}$ in the spectrum $\sigma_{r}\left(\vec{X}_{\delta}^{\mathbb{C}}\right)$ equals 0 or 1 , depending on the value of $r$. The computations are similar to those of Example 27 except in the case $r=3$, which generates multiplicity 3 . Since for $\Lambda_{3}$ the matrices $a_{\ell}$ are already lower-triangular, a coordinate change is not necessary, and it is easily seen that $s=3$ and

$$
\operatorname{ker}_{3} \vec{X}_{\delta}^{\mathbb{C}}=\mathbb{C} z_{1}^{2} z_{3}, \quad \operatorname{ker}_{3} \vec{X}_{\delta}^{\mathbb{C}(2)}=\mathbb{C}_{1} z_{2} z_{3}, \quad \operatorname{ker}_{3} \vec{X}_{\delta}^{\mathbb{C}(3)}=\mathbb{C} z_{2}^{2} z_{3} .
$$

The next two theorems follow from similar computations; no proofs are given. In both, the $Y_{i}$ are those defined in Theorem 4,

Theorem 29. If $a \neq 0$, the cohomology spaces of the structure $\Lambda_{3}$ are

$$
\begin{aligned}
& L H^{0 *}\left(\Re, \Lambda_{3}\right)=\mathbb{R}, \\
& L H^{1 *}\left(\Re, \Lambda_{3}\right)=\mathbb{R} Y_{1}+\mathbb{R} Y_{2}+\mathbb{R} Y_{3}, \\
& L H^{2 *}\left(\mathscr{R}, \Lambda_{3}\right)=\mathbb{R} Y_{23} \oplus \mathbb{R} Y_{31} \oplus \mathbb{R}\left(2 y z \partial_{31}+y^{2} \partial_{12}\right), \\
& L H^{3 *}\left(\mathscr{R}, \Lambda_{3}\right)=\mathbb{R} \partial_{123} \oplus \mathbb{R} y^{2} z \partial_{123} .
\end{aligned}
$$

Theorem 30. If $a \neq 0$, the cohomology spaces of the structure $\Lambda_{9}$ are where

$$
\begin{array}{lll}
L H^{0 *}\left(\mathscr{R}, \Lambda_{9}\right) & =\mathbb{R}, & L H^{2 *}\left(\mathscr{R}, \Lambda_{9}\right)=\bigoplus_{r \in \mathbb{N}} H_{r}^{2}, \\
L H^{1 *}\left(\mathscr{R}, \Lambda_{9}\right)=\mathbb{R} Y_{1}+\mathbb{R} Y_{2}+\mathbb{R} Y_{3}, & & L H^{3 *}\left(\mathscr{R}, \Lambda_{9}\right)=\bigoplus_{r \in \mathbb{N}} \mathbb{R} z^{r} \partial_{123},
\end{array}
$$

$$
\begin{aligned}
H_{0}^{2}= & \mathbb{R} \partial_{23}, \quad H_{1}^{2}=\mathbb{R} C_{1}^{0}, \quad H_{3}^{2}=\mathbb{R} C_{1}^{2}, \\
H_{2}^{2}= & \mathbb{R} x^{2} \partial_{23}+\mathbb{R} x z\left(\partial_{23}-2^{-1} \partial_{31}\right)+\mathbb{R}\left(x z \partial_{12}-z^{2} \partial_{23}\right) \\
& \quad+\mathbb{R}\left(y z \partial_{12}+\left(-27 a^{2} x^{2}-9 a x z+3 a y^{2}-z^{2}\right) \partial_{31}\right), \\
H_{r+1}^{2}= & \mathbb{R} C_{1}^{r}+\mathbb{R} C_{2}^{r} \quad \text { for } r \geq 3,
\end{aligned}
$$

with

$$
C_{1}^{r}=-a\left(x z^{r}+r y^{2} z^{r-1}\right) \partial_{12}+\left(9 a^{2} x y^{r}+a(3 r-1)(r+1)^{-1} z^{r+1}\right) \partial_{23}+a y z^{r} \partial_{31}
$$


and

$$
\begin{aligned}
& C_{2}^{r}=\left(9 a^{2} x y^{2} z^{r-2}-9 a r^{-1} x z^{r}\right. \\
& \left.+3 a(r-3)(r-1)^{-1} y^{2} z^{r-1}-3(r-1) r^{-1}(r+1)^{-1} z^{r+1}\right) \partial_{23} \\
& +\left(-a(r-2) y^{4} z^{r-3}+y^{2} z^{r-1}\right) \partial_{12} \\
& +\left(6 a(r-1)^{-1} x y z^{r-1}-a y^{3} z^{r-2}-r^{-1} y z^{r}\right) \partial_{31},
\end{aligned}
$$

and where the terms that contain a negative power of $x, y$, or $z$ are ignored.

\section{References}

[Ammar and Poncin 2008] M. Ammar and N. Poncin, "Formal Poisson cohomology of twisted $r$ matrix induced structures", Israel J. Math. 165 (2008), 381-411. MR 2009j:53104 Zbl 1146.53054

[Bolotnikov and Rodman 2002] V. Bolotnikov and L. Rodman, "Finite dimensional backward shift invariant subspaces of Arveson spaces", Linear Alg. Appl. 349 (2002), 265-282. MR 2003g:47042 Zbl 1019.46023

[Bourbaki 1975] N. Bourbaki, Éléments de mathématique, Fasc. XXXVIII: Groupes et algèbres de Lie, Chapitre VII: Sous-algèbres de Cartan, éléments réguliers. Chapitre VIII: Algèbres de Lie semi-simples déployées, Actualités Scientifiques et Industrielles 1364, Hermann, Paris, 1975. MR 56 \#12077 Zbl 0329.17002

[Brylinski 1988] J.-L. Brylinski, "A differential complex for Poisson manifolds", J. Differential Geom. 28:1 (1988), 93-114. MR 89m:58006 Zbl 0634.58029

[Dufour and Haraki 1991] J.-P. Dufour and A. Haraki, "Rotationnels et structures de Poisson quadratiques”, C. R. Acad. Sci. Paris Sér. I Math. 312:1 (1991), 137-140. MR 92a:53045 Zbl 0719.58001

[Evens et al. 1999] S. Evens, J.-H. Lu, and A. Weinstein, "Transverse measures, the modular class and a cohomology pairing for Lie algebroids", Quart. J. Math. Oxford Ser. (2) 50:200 (1999), 417436. MR 2000i:53114 Zbl 0968.58014

[Gammella 2002] A. Gammella, "An approach to the tangential Poisson cohomology based on examples in duals of Lie algebras", Pacific J. Math. 203:2 (2002), 283-320. MR 2003e:53131 Zbl 1055.53068

[Ginzburg 1999] V. L. Ginzburg, "Equivariant Poisson cohomology and a spectral sequence associated with a moment map", Internat. J. Math. 10:8 (1999), 977-1010. MR 2001g:53143 Zbl 1061. 53059

[Grabowski and Marmo 2003] J. Grabowski and G. Marmo, "The graded Jacobi algebras and (co)homology”, J. Phys. A 36:1 (2003), 161-181. MR 2003m:53144 Zbl 1039.53090

[Grabowski et al. 1993] J. Grabowski, G. Marmo, and A. M. Perelomov, "Poisson structures: Towards a classification”, Modern Phys. Lett. A 8 (1993), 1719-1733. MR 94j:58063 Zbl 1020.37529

[Huebschmann 1990] J. Huebschmann, "Poisson cohomology and quantization", J. Reine Angew. Math. 408 (1990), 57-113. MR 92e:17027 Zbl 0699.53037

[Huebschmann 1999] J. Huebschmann, "Duality for Lie-Rinehart algebras and the modular class", J. Reine Angew. Math. 510 (1999), 103-159. MR 2000f:53109 Zbl 1034.53083

[Ibáñez et al. 2001] R. Ibáñez, M. de León, B. López, J. C. Marrero, and E. Padrón, "Duality and modular class of a Nambu-Poisson structure", J. Phys. A 34 (2001), 3623-3650. MR 2002e:53127 Zbl 1021.53060 
[Koszul 1950] J.-L. Koszul, "Homologie et cohomologie des algèbres de Lie", Bull. Soc. Math. France 78 (1950), 65-127. MR 12,120g Zbl 0039.02901

[Koszul 1985] J.-L. Koszul, "Crochet de Schouten-Nijenhuis et cohomologie”, pp. 257-271 in The mathematical heritage of Élie Cartan (Lyon, 1984), Société Mathématique de France, Paris, 1985. MR 88m:17013 Zbl 0615.58029

[Koszul 1994] J.-L. Koszul, Selected papers of J.-L. Koszul, Series in Pure Mathematics 17, World Scientific, River Edge, NJ, 1994. MR 95f:01037 Zbl 0862.01036

[de León et al. 1997] M. de León, J. C. Marrero, and E. Padrón, "Lichnerowicz-Jacobi cohomology”, J. Phys. A 30:17 (1997), 6029-6055. MR 98k:58099 Zbl 0951.37033

[de León et al. 2003] M. de León, B. López, J. C. Marrero, and E. Padrón, "On the computation of the Lichnerowicz-Jacobi cohomology”, J. Geom. Phys. 44:4 (2003), 507-522. MR 2003m:53145 Zbl 1092.53060

[Lichnerowicz 1977] A. Lichnerowicz, "Les variétés de Poisson et leurs algèbres de Lie associées", J. Differential Geometry 12:2 (1977), 253-300. MR 58 \#18565 Zbl 0405.53024

[Liu and Xu 1992] Z. J. Liu and P. Xu, "On quadratic Poisson structures", Lett. Math. Phys. 26:1 (1992), 33-42. MR 93k:58097 Zbl 0773.58007

[Manchon et al. 2002] D. Manchon, M. Masmoudi, and A. Roux, "On quantization of quadratic Poisson structures”, Comm. Math. Phys. 225:1 (2002), 121-130. MR 2002k:53181 Zbl 1002.53060

[Masmoudi and Poncin 2007] M. Masmoudi and N. Poncin, "On a general approach to the formal cohomology of quadratic Poisson structures", J. Pure Appl. Algebra 208:3 (2007), 887-904. MR 2007m:17030 Zbl 05083168

[Monnier 2001] P. Monnier, "Computations of Nambu-Poisson cohomologies", Int. J. Math. Math. Sci. 26:2 (2001), 65-81. MR 2002d:53112 Zbl 1103.53050

[Monnier 2002a] P. Monnier, "Formal Poisson cohomology of quadratic Poisson structures", Lett. Math. Phys. 59:3 (2002), 253-267. MR 2003c:53122 Zbl 1010.53059

[Monnier 2002b] P. Monnier, "Poisson cohomology in dimension two", Israel J. Math. 129 (2002), 189-207. MR 2003h:53117 Zbl 1077.17018

[Nakanishi 1997] N. Nakanishi, "Poisson cohomology of plane quadratic Poisson structures", Publ. Res. Inst. Math. Sci. 33:1 (1997), 73-89. MR 98d:58063 Zbl 0970.53042

[Nakanishi 2006] N. Nakanishi, "Computations of Nambu-Poisson cohomologies: case of NambuPoisson tensors of order 3 on $\mathbb{R}^{4 ",}$, Publ. Res. Inst. Math. Sci. 42 (2006), 323-359. MR 2007k:53135 Zbl 1135.53060

[Pichereau 2005] A. Pichereau, "Cohomologie de Poisson en dimension trois", C. R. Math. Acad. Sci. Paris 340:2 (2005), 151-154. MR 2006b:17036 Zbl 1070.53051

[Pichereau and Van de Weyer 2008] A. Pichereau and G. Van de Weyer, "Double Poisson cohomology of path algebras of quivers", J. Algebra 319:5 (2008), 2166-2208. MR 2009b:17053 Zbl 05271126

[Roger and Vanhaecke 2002] C. Roger and P. Vanhaecke, "Poisson cohomology of the affine plane", J. Algebra 251:1 (2002), 448-460. MR 2003g:17031 Zbl 0998.17023

[Roytenberg 2002] D. Roytenberg, "Poisson cohomology of SU(2)-covariant "necklace" Poisson structures on $S^{2}$ ", J. Nonlinear Math. Phys. 9:3 (2002), 347-356. MR 2003h:53118

[Vaisman 1990] I. Vaisman, "Remarks on the Lichnerowicz-Poisson cohomology", Annales Inst. Fourier (Grenoble) 40:4 (1990), 951-963 (1991). MR 92c:58155 Zbl 0708.58010

[Vaisman 1994] I. Vaisman, Lectures on the geometry of Poisson manifolds, Progress in Mathematics 118, Birkhäuser, Basel, 1994. MR 95h:58057 Zbl 0810.53019 
[Xu 1992] P. Xu, "Poisson cohomology of regular Poisson manifolds", Ann. Inst. Fourier (Grenoble) 42:4 (1992), 967-988. MR 94d:58167 Zbl 0759.58020

[Xu 1999] P. Xu, "Gerstenhaber algebras and BV-algebras in Poisson geometry", Comm. Math. Phys. 200:3 (1999), 545-560. MR 2000b:17025 Zbl 0941.17016

Received January 20, 2009. Revised May 29, 2009.

MOURAD AMMAR

INSTITUTE OF MATHEMATICS

UNIVERSITY OF LUXEMBOURG, CAMPUS LIMPERTSBERG

162A, AVENUE DE LA FAÏ̈NCERIE

L-1511 LUXEMBOURG CITY

LUXEMBOURG

mourad.ammar@uni.lu

GUY KASS

INSTITUTE OF MATHEMATICS

UNIVERSITY OF LUXEMBOURG, CAMPUS LIMPERTSBERG

162A, AVENUE DE LA FAÏENCERIE

L-1511 LUXEMBOURG CITY

LUXEMBOURG

guy.kass@uni.lu

MOHSEn MASMOUdi

UNIVERSITÉ HENRI POINCARÉ

INSTITUT ELIE CARTAN

B.P. 239

F-54 506 VAndoeuvre-Les-NANCy CedeX

FRANCE

Mohsen.Masmoudi@iecn.u-nancy.fr

\section{NORBERT PONCIN}

InstiTUTE OF MATHEMATICS

UNIVERSITY OF LUXEMBOURG, CAMPUS LIMPERTSBERG

162A, AVENUE DE LA FAÏENCERIE

L-1511 LUXEMBOURG CITY

LUXEMBOURG

norbert.poncin@uni.lu

http://wwwen.uni.lu/research/fstc/unite_de_recherche_en_mathematiques/research/ 